\title{
System-Level Sensitivity Analysis of SiNW-bioFET-Based Biosensing Using Lockin Amplification
}

Patou, François; Dimaki, Maria; Kjærgaard, Claus; Madsen, Jan; Svendsen, Winnie Edith

Published in:

I E E E Sensors Journal

Link to article, DOI:

10.1109/JSEN.2017.2742018

Publication date:

2017

Document Version

Peer reviewed version

Link back to DTU Orbit

Citation $(A P A)$ :

Patou, F., Dimaki, M., Kjærgaard, C., Madsen, J., \& Svendsen, W. E. (2017). System-Level Sensitivity Analysis of SiNW-bioFET-Based Biosensing Using Lockin Amplification. I E E E Sensors Journal, 17(19), 6295-6311. https://doi.org/10.1109/JSEN.2017.2742018

\section{General rights}

Copyright and moral rights for the publications made accessible in the public portal are retained by the authors and/or other copyright owners and it is a condition of accessing publications that users recognise and abide by the legal requirements associated with these rights.

- Users may download and print one copy of any publication from the public portal for the purpose of private study or research.

- You may not further distribute the material or use it for any profit-making activity or commercial gain

- You may freely distribute the URL identifying the publication in the public portal 


\title{
System-level Sensitivity Analysis of SiNW-bioFET-based Biosensing Using Lock-in Amplification
}

\author{
François Patou, Maria Dimaki, Claus Kjærgaard, Jan Madsen, and Winnie E. Svendsen
}

\begin{abstract}
Although Silicon Nanowire biological Field-Effect Transistors (SiNW-bioFETs) have steadily demonstrated their ability to detect biological markers at ultra-low concentration, they have not yet translated into routine diagnostics applications. One of the challenges inherent to the technology is that it requires an instrumentation capable of recovering ultra-low signal variations from sensors usually designed and operated in a highly-resistive configuration. Often overlooked, the SiNWbioFET/instrument interactions are yet critical factors in determining overall system biodetection performances.

Here, we carry out for the first time the system-level sensitivity analysis of a generic SiNW-bioFET model coupled to a custom-design instrument based on the lock-in amplifier. By investigating a large parametric space spanning over both sensor and instrumentation specifications, we demonstrate that systemwide investigations can be instrumental in identifying the design trade-offs that will ensure the lowest Limits-of-Detection. The generic character of our analytical model allows us to elaborate on the most general SiNW-bioFET/instrument interactions and their overall implications on detection performances. Our model can be adapted to better match specific sensor or instrument designs to either ensure that ultra-high sensitivity SiNW-bioFETs are coupled with an appropriately sensitive and noise-rejecting instrumentation, or to best tailor SiNW-bioFET design to the specifications of an existing instrument.
\end{abstract}

Index Terms-SiNW-bioFET; System analysis; Diagnostics; Instrumentation; Lock-in amplification

\section{INTRODUCTION}

Silicon-Nanowire biological Field-Effect Transistors (SiNW-bioFETs) were introduced in 2001 as a promising solution for the real-time, low-cost, label-free, high throughput analysis of a variety of chemical and biological markers present at low-concentration in low sample processing volumes [1]. They have since demonstrated their potential in a wide variety of applications ranging from the quantification of metabolites [2], or nucleic acids [3]-[5], to the detection of myriad proteins [6] and viruses [7], [8]. For all these applications Limits of detection (LoD) in the picomolar range are now common while femtomolar LoDs have also

Post-doctoral researcher François Patou is with the Engineering Systems Division (ESD) at the Department of Management Engineering, Technical University of Denmark, Lyngby, (frpato@dtu.dk)

Senior researcher Maria Dimaki is with the Department of Micro- and Nanotechnology, Technical University of Denmark, Lyngby

Professor Claus Kjærgaard is with the Department of Electrical Engineering, Technical University of Denmark

Professor Jan Madsen is with the Department of Applied Mathematics and Computer Science, Technical University of Denmark, Lyngby

Associate Professor Winnie E. Svendsen is with the Department of Microand Nanotechnology, Technical University of Denmark, Lyngby recently become a reality [9]-[11]. Such low LoDs can be reached by the fine tuning of static design parameters such as device dimensionality, and by carefully choosing the dynamic operation point at which measurements should be done. Several groups have already thoroughly documented the benefits of small cross-sectional SiNW channels or of biasing the sensor in the subthreshold regime [12], [13]. Although these investigations and experimental validation studies are sufficiently informative for many applications, they are limited in addressing the likely scenario of the integration of SiNWbioFETs in a custom-built diagnostics system such as the one we introduced in [14]. Indeed, the SiNW-bioFET design and operation specifications that promise the greatest LoD performances are also those that are the most challenging from an instrumentation perspective. The ultra-narrow channels and subthreshold regime operation advocated for best sensitivity indeed require the measurement of extremely low deviations from the device baseline conductance. This, in turns, means that the reliable detection or quantification of the smallest signal variations generated by the binding of few target analytes at the gate will necessitate the accurate recovery of challengingly low input signal changes.

Several groups have turned towards CMOS technology to address these instrumentation challenges since integrated analog front-end amplification can deliver unmatched signal conditioning performances. CMOS analog integration is also preferred for interfacing massively paralleled sensing structures, as often needed in applications involving highly-multiplexed detection schemes. Full analog-CMOS/SiNW-bioFET integration was achieved recently [15]. Although such integrated hybrid-systems may rid themselves of many instrumentation issues, modular configurations, where sensor and instrument are not tightly coupled, are still predominant. One reason for this is the common requirement for a variety of instrumentation techniques and settings to operate individual or mixedbiosensing technologies. Susloparova et al., for instance, recently used impedance spectroscopy to instrument an ionsensitive FET in order to investigate single-cell adhesion and detachment processes [16]. In this scenario the flexibility of a versatile modular instrumentation was likely preferred over the development of a costly Application Specific Integrated Circuit that would provide the same functionality.

For such non-integrated SiNW-bioFET/instrument systems, noise and offsets become concerns. Both extrinsic noise sources and the instrumentation noise itself threaten to deteriorate the integrity SiNW-bioFETs signals and to cancel 


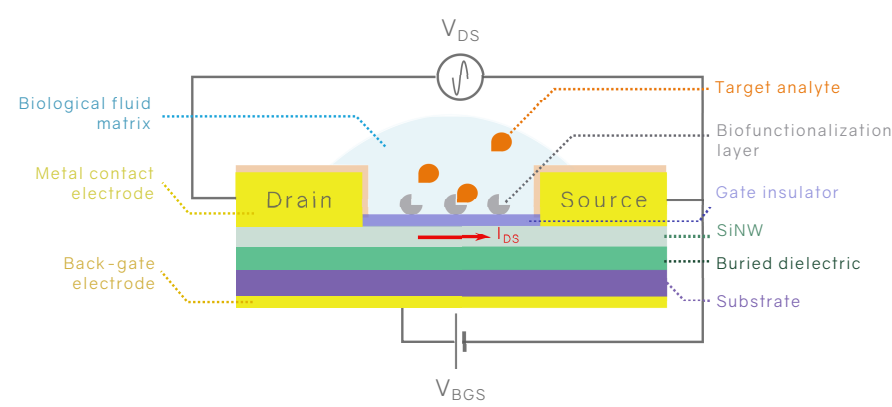

(a)

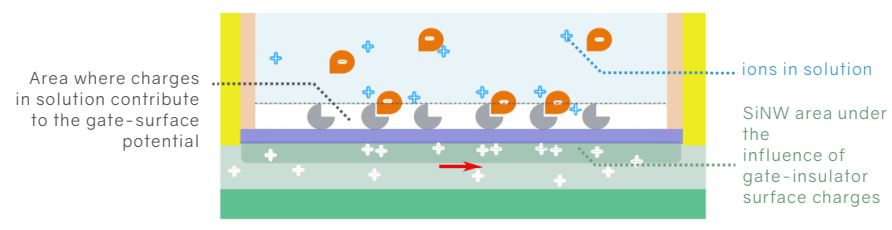

(b)

Fig. 1. a - Conventional architecture of a SiNW-bioFET. $\mathrm{b}$ - Illustration of the Thomas-Fermi and Debye screening length

the benefits to avail high-sensitivity biosensors altogether. The following investigation pioneers the system-level analysis of the effect of SiNW-bioFET/instrumentation couplings, and in particular the effect of intrinsic instrumentation noise, on systemic LoD performances. Looking specifically at the lock-in amplification technique, our endeavour illustrates the importance of leading system-wide investigations in order to ensure that ultra-high SiNW-bioFET sensitivity is leveraged by an appropriately sensitive and noise-rejecting instrumentation, or in order to best tailor SiNW-bioFET design to the specifications of an existing instrumentation.

\section{SYSTEM DESCRIPTION}

\section{A. The SiNW-bioFET}

1) Generalities: Largely inspired from the physical principles and design of the metal-oxide semiconductor FET (MOSFET), SiNW-bioFETs allow the detection of biological species in solution by harnessing the field effect produced by the electrical charges of targeted molecules selectively binding to the gate of the device. A common planar SiNW-bioFET design is illustrated in figure 1. The adoption of specific gate surface chemistries is necessary in order to ensure selectivity towards the target of interest. The overall charge accumulated at the gate surface may either deplete the semiconducting SiNW channel from its majority carriers or induce their accumulation depending on its sign and distribution. Although recent findings have highlighted the potential of the memresistive effects of SiNW-FETs in biosensing applications [17], most common characterizations involve measuring the variation in SiNW carrier concentration by proxy of measurable changes in SiNW conductivity using electrical IV instrumentation techniques.

2) Sensitivity: SiNW-bioFET sensitivity and Limit of Detection (LoD) are determined by a number of interrelated factors, including SiNW geometry and the SiNW concentration of dopant impurities. Generally, smaller SiNW cross-sectional areas and lower dopant concentrations will facilitate variations in gate-surface charges to translate into larger drain-source current fluctuations, and hence higher sensitivities [18]-[22]. That is essentially because smaller SiNW cross-sectional areas may allow surface charges at the gate to influence SiNW charge carriers throughout a greater proportion of their semiconducting pathway. SiNW length does usually not have any effect on biodetection sensitivity as SiNW-bioFET channels are junction-less, and often significantly longer than CMOS transistors channels. SiNW-bioFETs therefore rarely suffer from the "short-channel effects"that challenge the design of CMOS transistors, in particular the trading-off of transistor size, speed and behaviour predictability.

The SiNW-bioFET model on which we elaborate throughout the rest of this study is that of De Vico et al. [23]. The device presents a circular cross-sectional channel as illustrated in figure 2. This geometry has the merit of simplifying the analytical investigation of the sensors physico-chemical interactions with its environment. We discuss the implications of considering an ideal circular cross-section channel in section $\mathrm{V}$.

We consider a silicon channel of length $L=20 \mu \mathrm{m}$, and radius $R=20 \mathrm{~nm}$. The SiNW is homogeneously p-doped with boron acceptor ions originally specified with a concentration $N_{\mathrm{A}}=1 \times 10^{24} \mathrm{~m}^{-3}$. In an unbiased state, we assume that the carrier concentration within the channel remains at its intrinsic value $N_{\mathrm{A}}$. The carriers have a mobility $\mu=2 \mathrm{~cm}^{2} / \mathrm{V} \mathrm{s}$

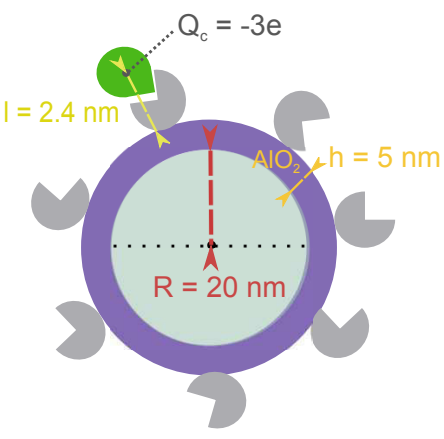

Fig. 2. Theoretical circular SiNW-bioFET channel cross-section.

Nair et al. were among the first to investigate comprehensively the influence of device geometry on sensing performances [20]. They also demonstrated that decreasing SiNW dopant concentration could benefit SiNW-bioFET sensitivity until a certain level, beyond which inherent statistical fluctuations in the low discrete number of dopant ions in each channel would result in unacceptable behavioral inhomogeneity between several SiNW-bioFETs on the same substrate. Buitrago et al. later showed that the sensitivity of SiNWs presenting rectangular cross-sections scaled down to $10 \mathrm{~nm} \times 10 \mathrm{~nm}$ was independent from dopant concentrations [24], findings that were supported by Elfstöm et al.'s experimental work [25]. The influence of dopant concentration on sensitivity is a consequence of the Thomas-Fermi screening phenomenon (figure 1b): charges borne by the dopant ions in the semiconductor screen the electrical field generated by the charges at the gate insulator surface. This phenomenon is quantified by a length metric, representing the distance from the silicon surface to the bulk of the material beyond which the electrical field does not influence the charge carriers anymore. For dopant 
concentrations inferior to $1 \times 10^{19} \mathrm{~cm}^{-3}$, this metric is better approximated by the Debye-Hückel theory. An expression of the Debye-Hückel screening -or carrier screening- length $\lambda_{D_{\mathrm{SiNW}}}$ for the silicon wire is then given by:

$$
\lambda_{D_{\mathrm{SiNW}}}=\sqrt{\frac{\epsilon_{S i} K T}{p e^{2}}},
$$

where $p$ is the dopant concentration, $\epsilon_{\mathrm{Si}}$ is the permittivity of silicon, $K$ is the Boltzmann constant, $T$ the temperature in Kelvin and $e$ is the elementary charge.

Although essential, the consideration of geometry and dopant concentration does not alone allow the prediction of the system-level LoD as the latter also depends on the physical laws binding SiNW properties to the properties of the solution in which they are immersed. In [20], Nair et al. considered the effect of the ionic-strength of the solution on sensitivity. This parameter plays a prominent role in the definition of the Debye-Hückel screening length of the solution: the metric translating the relation between the ionic strength of the solution in contact with the gate with the effective distance beyond which the charges borne by the molecules of interest in solution are not contributing to the surface potential at the gate-liquid interface (equation 2). Charged analyte molecules beyond $\lambda_{D_{\text {sol }}}$ will be screened by surrounding ions in solution, and will not contribute to building up the surface potential effectively modulating the SiNW conductance (figure 1b). Nair et al. derived important recommendations from these findings, advocating SiNW cross-sectional dimensions in the range or inferior to the Debye-Hückel screening length in order to promote efficient sensing. [18]-[20], [22].

$$
\lambda_{D_{\mathrm{sol}}}=\sqrt{\frac{\epsilon_{0} \epsilon_{s o l} K T}{2 N_{A} e^{2} I}}
$$

3) Gate properties: The gate properties of SiNW-bioFETs are key in defining device sensitivity to a particular molecular target. Common to both MOS-FETs and SiNW-bioFETs, the thin dielectric layer effectively gating the channel will, for the latter technology, screen surfaces charges accumulating at the gate-liquid interface. Reducing the thickness of the dielectric layer should allow target charges to influence the channel carriers more effectively [26], a potential optimization strategy yet compromised by the difficulty of reliably and reproducibly reaching single-nanometric gate thicknesses using conventional microfabrication methods.

The functional specificity of the SiNW-bioFET comes from the biofunctionalization layer coating the surface of the gate. Numerous strategies for the functionalization of the bare silicon surface of the SiNW or most often of the thin oxide layer wrapping the SiNW present as many opportunities for sensing performance optimisation. Specific bio-conjugation mechanisms between gate-receptor molecules and their target analytes, the spatial alignment of the receptor probes or the use of charged reporter molecules are examples of possible amelioration schemes, all of which can be combined in search of greater performances still. An extensive review of experimental research on these topics is available in [27]. De Vico et al. were the first to investigate the most significant properties of the biofunctional layer in their computational model [13]. They succeeded in doing so by specifying a series of abstracted parameters helping to circumvent the intricacy of considering the complex laws and behaviours governing the interactions between the various biological elements of the system. More specifically, they abstracted the linker-receptorligand geometries by specifying a point-charge of value $Q_{c}$ for their molecular complex and the distance $l$ between that point-charge and the gate-insulator surface (figure 2). They finally took into account the density of biological receptors bound to the gate surface: the surface coverage $s_{d}$, as well as the dissociation constant $K_{d}$ of the specific receptor/ligand complex. $K_{d}$ determines $\theta$ : the fraction of adsorbed ligand at the surface of the gate as a function of the concentration of ligand molecules in solution so that:

$$
\theta=\frac{[\text { ligand }]}{[\text { ligand }]+K_{d}}
$$

These latter properties subsequently allow the computation of the average cumulative surface charge $N_{\text {cplx }}$ covering the gate once biological steady-state is reached. We will, for the rest of this study, consider a biofunctional coating made of ABL-tyrosine kinase receptors, specific to the ATP ligand molecule, pillar of cell metabolism. Like De Vico et al., we abstract the ABL/ATP receptor-ligand complex to a single charge $Q_{c}=-3 e$, at a distance of $l=2.4 \mathrm{~nm}$ from the gate surface. We assume that $Q_{c}$ is entirely borne by the ATP molecule and thus that variations in coverage of the SiNW-bioFET gate surface by ABL-tyrosine kinase receptor molecules will not alter the intrinsic resistance of the SiNW. The dissociation constant of the ATP/ABL-tyrosine kinase complex is $K_{d}=62.9 \mathrm{nmol} / \mathrm{L}$.

The gate was assumed to be made of $h=5 \mathrm{~nm}$ of aluminium dioxide $\left(\varepsilon_{\mathrm{ox}}=9.34\right)$ and the ionic strength of the surfacing solution was fixed at $1 \times 10^{-12} \mathrm{~mol} \mathrm{~m}^{-3}$. The surface receptors (ABL) density $s_{d}$, at the gate was set at $65 \times 10^{12}$ receptors $/ \mathrm{m}^{2}$ according to results from ongoing surface functionalization experimental studies. This assumption enabled us to approximate the number of receptor molecules $N_{\text {rcp }}$ covering the entire surface area of the gate oxide $A_{\text {gate }}$ by:

$$
\begin{gathered}
N_{\text {rcp }} \approx s_{d} \times A_{\text {gate }} \\
N_{\text {rcp }} \approx 65 \times 10^{12} \times L \times(2 \pi(R+h))
\end{gathered}
$$

By defining the average cross-sectional surface area of the ligand/receptor complex $A_{\text {cplx }}$, we may derive an approximation of the maximum number of receptor/ligand complexes at the gate $N_{\text {cplx.max }}$, so that:

$$
N_{\text {cplx.max }}=\min \left(N_{\text {rcp }}, \frac{A_{\text {gate }}}{A_{\text {cplx }}}\right)
$$

Should the size of the ligand be greatly superior to that of the receptor, then the maximum number of ligand/receptor complexes at the gate may be limited by the space necessary for these complexes to form. 
From $N_{\text {cplx.max }}$ we can calculate the approximate number of complexes $N_{\text {cplx }}$ present at the gate using the adsorbed fraction $\theta$. We obtain:

$$
N_{\text {cplx }}=N_{\text {cplx.max }} \times \theta
$$

The overall surface charge density $\sigma_{b}$, corresponding to the average charge per unit of surface area from all ligand/receptor complexes bound to the gate can then be calculated as:

$$
\begin{gathered}
\sigma_{b}=\frac{N_{\mathrm{cplx}} \times Q_{c}}{A_{\text {gate }}} \\
\sigma_{b}=\frac{N_{\mathrm{cplx}} \times Q_{c}}{L \times(2 \pi(R+h+l))}
\end{gathered}
$$

From De Vico et al., the relative change in SiNW channel conductance can then be calculated as:

$$
\frac{\Delta G}{G 0}=-\frac{2}{R e p_{0}} \Gamma\left(\Gamma_{l} \sigma_{b}+\sigma_{s}\right)
$$

$$
\begin{gathered}
\Gamma=\frac{\varepsilon_{\mathrm{Si}} K_{0}(B) \frac{\lambda_{D_{\mathrm{sol}}}}{\lambda_{D_{\mathrm{SiNW}}}} I_{1}\left(\frac{R}{\lambda_{D_{\mathrm{SiNW}}}}\right)}{\left[K_{0}(B)\left(\frac{1}{B}\right)+\ln \left(\frac{R+h}{R}\right) K_{1}(B) \frac{\varepsilon_{\mathrm{Sol}}}{\varepsilon_{\mathrm{ox}}}\right] \varepsilon_{\mathrm{Si}}\left(\frac{R}{\lambda_{D_{\mathrm{SiNW}}}}\right) I_{1}\left(\frac{R}{\lambda_{D_{\mathrm{SiNW}}}}\right)+\varepsilon_{\mathrm{Sol}} K_{1}(B) I_{0}\left(\frac{R}{\lambda_{D_{\mathrm{SiNW}}}}\right)} \\
\text { with: } B=\frac{R+h}{\lambda_{D_{\mathrm{sol}}}}
\end{gathered}
$$

We will assume $\sigma_{s}$, the surface charge density directly at the gate surface (e.g. from trapped charges), to be null. $R$ represents the radius of the SiNW. $p_{0}$ corresponds to the dopant concentration $N_{A}$ and $e$ is the elementary charge. The parameter $\Gamma$ translates the sensitivity of the device according to equation 11, with $I_{0}, I_{1}, K_{0}, K_{1}$ the modified Bessel functions of the first and second kind; $\varepsilon_{\mathrm{Si}}, \varepsilon_{\mathrm{ox}}, \varepsilon_{\mathrm{Sol}}$ stand for the relative permittivities of the SiNW, the aluminium gate oxide and the solution respectively [13], [28]. Finally, $\Gamma_{l}$ is a dimensionless parameter quantifying the effect of $\sigma_{b}$ and is defined by:

$$
\Gamma_{l}=2 \frac{R}{R+l}\left(1+\sqrt{\frac{R}{R+l}} \exp \left(l / \lambda_{D_{\mathrm{Sol}}}\right)\right)^{-1}
$$

If the amplitude of the potential applied between source and drain of the sensor is known then we can calculate the current $I_{\mathrm{DS}}$ flowing through the SiNW channel:

$$
I_{\mathrm{DS}}=I_{\mathrm{DS}_{0}}\left(1-2 \Gamma\left(\frac{\Gamma_{l} \sigma_{b}+\sigma_{s}}{H e N_{A}}\right)\right)
$$

$I_{\mathrm{DS}_{0}}$ can be determined in an unbiased transistor state, when no influence is assumed from charged species in the sample solution. The carrier concentration within the channel can then be assimilated to its intrinsic value $N_{\mathrm{A}}$. Ohm's law and Drude's model of conductivity allow us to specify the SiNW's intrinsic resistance which in our case can be calculated with $R=20 \mathrm{~nm}$ as:

$$
\begin{gathered}
R_{\mathrm{SiNW}}=\frac{1}{\sigma} \cdot \frac{L}{\pi R^{2}} \\
R_{\mathrm{SiNW}}=\frac{1}{\mu \cdot e \cdot N_{\mathrm{A}}} \cdot \frac{L}{\pi R^{2}},\left(e=1.602 \times 10^{-19} \mathrm{C}\right) \\
R_{\mathrm{SiNW}} \simeq 4.967 \times 10^{8} \Omega
\end{gathered}
$$

The intrinsic current value $I_{\mathrm{DS}_{0}}$ becomes:

$$
I_{\mathrm{DS}_{0}}=V_{\mathrm{DS}} \cdot \mu \cdot e \cdot N_{\mathrm{A}} \frac{\pi R^{2}}{L}
$$

\section{B. Signal conditioning}

We consider a SiNW-bioFET as specified in the previous section. We intend to measure the steady-state SiNW-bioFET current deviation from its baseline value, upon exposure of the gate to a solution containing ATP, using Phase-Sensitive detection (PSD). PSD, also called lock-in amplification, is an AC-measurement technique used in many scientific and engineering contexts necessitating the recovery of signals buried in noise. We elaborate on the elementary principle of PSD later in this section.

As the intrinsic resistance of our SiNW-bioFET may be high, the use of an $\mathrm{AC}$-measurement method requires the consideration of the shunt capacitance $C_{\text {shunt }}$, the equivalent capacitance linking the instrumentation input and output, formed by the network of capacitive components characterising the sensor and its substrate (figure 3). These parasitic capacitive components may result from poor sensor design or simply from the non-idealities of the materials and processes used 
for device fabrication. For highly resistive SiNW channels, $C_{\text {shunt }}$ will limit the frequency range over which the SiNW resistance can be measured. The complex impedance of the $R_{\text {SiNW }} / C_{\text {shunt }}$ network is given by:

$$
Z_{\text {sens }}(\omega)=\frac{R_{\mathrm{SiNW}}}{1+j \omega C_{\text {shunt }} R_{\mathrm{SiNW}}}
$$

A normalized magnitude Bode plot of the $R_{\mathrm{SiNW}} / C_{\text {shunt }}$ network (i.e. $20 \log \left(\left|Z_{\text {sens }}\right| / R_{\text {SiNW }}\right)$ ) and phase $\angle Z_{\text {sens }}$ for $C_{\text {shunt }}=100 \times 10^{-15} \mathrm{~F}$ and $R_{\mathrm{SiNW}}=4.967 \times 10^{8} \Omega$ is presented in figure 4 . The value for $C_{\text {shunt }}$ was set to an arbitrary yet realistic estimate of what a the shunt capacitance of a SiNW-bioFET patterned on a silicon oxide substrate may be. For excitation frequencies beyond the cutoff $f_{\text {sens }}=1 / 2 \pi R_{\text {SiNW }} C_{\text {shunt }} \simeq 3.204 \times 10^{3} \mathrm{~Hz}$, the impedance network magnitude rolls off at $20 \mathrm{~dB} /$ decade, limiting the ACmeasurements of the SiNW-bioFET currents to frequencies significantly inferior to $f_{\text {sens }}$.

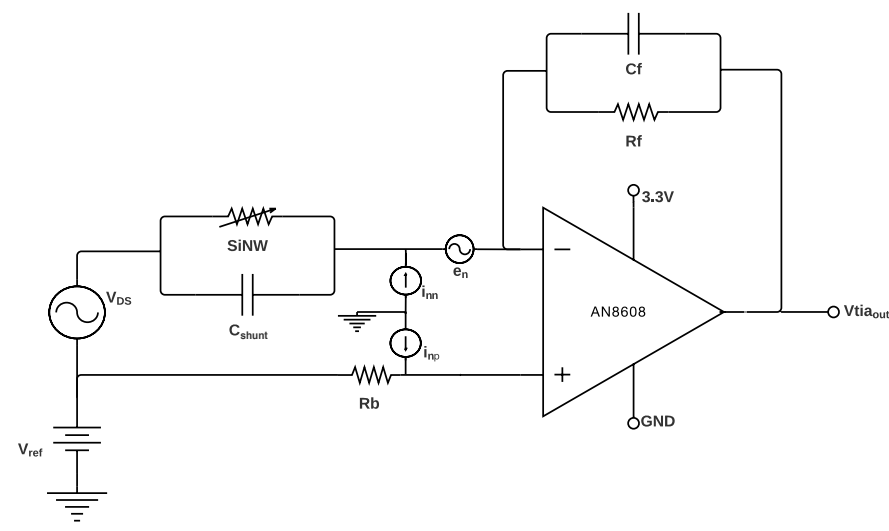

Fig. 3. Transimpedance amplification of the drain-source current $I_{\mathrm{DS}}$ flowing through a SiNW-bioFET. This electric model takes into account the input current noise $i_{n}=i_{n n}=i_{n p}$ of the amplifier and its input voltage noise $e_{n}$. These intrinsic noise components are characterized by their Power Spectral Density (PSD). Various noise harmonics will be modulated differently, depending on the transfer function $H_{\text {TIA }}(\omega)$ of the TIA.

We now consider the signal conditioning circuitry connecting the sensor to the PSD module (figure 3). The analog front-end of this block is composed of a single-stage voltage feedback transimpedance amplifier (TIA) meant to collect and amplify the SiNW-bioFET drain-source current $I_{\mathrm{DS}}$, converting it at the TIA output into the voltage signal $V_{\text {TIA }_{\text {out }}}$ [29]. The TIA is followed by a passive RC circuit meant to drive the Analog to Digital Converter marking the input of the PSD stage.

Although various extrinsic noise sources may corrupt signal integrity along the signal acquisition chain, we assume from now on the sole contribution of intrinsic noise i.e. noise originating from the signal-conditioning block components themselves.

1) Transimpedance amplification stage: For highimpedance applications such as the measurement of SiNW-bioFET currents biased in the subthreshold regime, the sensor shunt capacitance $C_{\text {shunt }}$ will not only determine the operating point of the sensor but it should also be considered for specifying the feedback network and hence the configuration of the TIA. Indeed, $C_{\text {shunt }}$ adds in parallel with the common-mode capacitance of the TIA inputs $C_{\mathrm{cm}}$. The TIA's transimpedance transfer function $H_{\mathrm{TIA}}(\omega)$ can then be defined as:

$$
H_{\mathrm{TIA}}(\omega)=\frac{V_{\mathrm{TIA}_{\text {out }}}}{I_{\mathrm{TIA}_{\text {in }}}}=\frac{Z_{f}}{1+\frac{1}{A_{0}(\omega) \beta(\omega)}}
$$

, with according to control theory:

$$
\left\{\begin{array}{l}
A_{0}(\omega)=\frac{1}{1+j \frac{\omega}{\omega_{0}}} \\
\beta(\omega)=\frac{Z_{\mathrm{sens}}}{Z_{\mathrm{sens}}+Z_{f}}=\frac{1+j \frac{\omega}{\omega_{f}}}{1+j \frac{\omega}{\omega_{i+f}}}
\end{array}\right.
$$

$\left\{\begin{array}{l}\omega_{0} \text { is the amplifier's open-loop gain cut-off frequency } \\ \omega_{f}=1 / R_{f} C_{f} \\ \omega_{i+f}=1 / R_{f}\left(C_{i}+C_{f}\right), C_{i}=C_{\text {shunt }}+C_{\mathrm{cm}}\end{array}\right.$

$\left\{\begin{array}{l}f_{0}=\omega_{0} / 2 \pi \\ f_{f}=\omega_{f} / 2 \pi \\ f_{i+f}=\omega_{i+f} / 2 \pi\end{array}\right.$

The cutoff frequency $f_{f}$ corresponds to the frequency marking the beginning of the roll-off of the TIA's signal gain profile (green curve in figure 5). $f_{f}$ should set the ultimate upper excitation frequency limit for the operation of the TIA. The practical maximum bandwidth for $\mathrm{AC}$ operation of a high-impedance SiNW-bioFET interfaced to this TIA is thus restricted to the range in which both sensor and amplifier operate in their flat-band region or $f_{\max }=\min \left(f_{f}, f_{\text {sens }}\right)$.

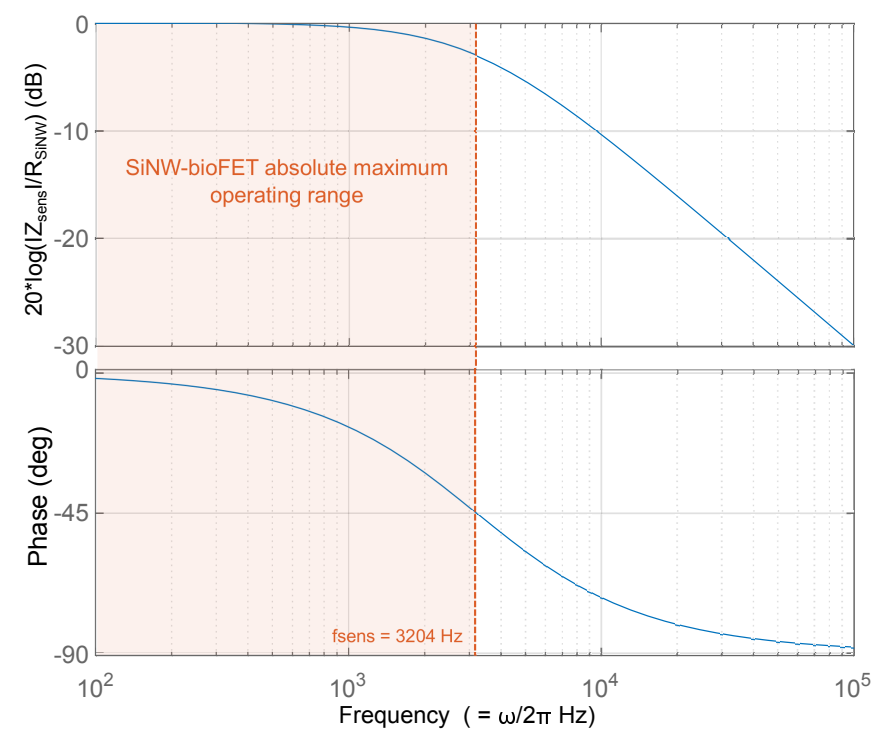

Fig. 4. Normalized impedance magnitude and phase Bode plot of a SiNWbioFET and its shunt capacitance. The operating range for the sensor spans from DC to a frequency that must remain significantly smaller than the cutoff frequency $f_{\text {sens }}$, with $f_{\text {sens }}=1 / 2 \pi R_{\text {SiNW }} C_{\text {shunt }} \simeq 3204 \mathrm{~Hz}$ if $R_{\text {SiNW }}=$ $4.967 \times 10^{8} \Omega$ and $C_{\text {shunt }}=100 \times 10^{-15} \mathrm{~F}$ 
Our TIA stage is built around the AN8608 quad amplifier [30]. The specifications of both the SiNW-bioFET discussed throughout the previous section and those of the AN8608 allow the analysis of the TIA transfer function $H_{\text {TIA }}(\omega)$. The closedloop magnitude Bode plot of $H_{\mathrm{TIA}}(\omega)$ for $R_{f}=1 \mathrm{G} \Omega$ and $C_{f}=1.5 \mathrm{pF}$ is presented in figure 5 . The cutoff frequency $f_{f}$ is limited to $f_{f}=1 / 2 \pi R_{f} C_{f}=106 \mathrm{~Hz}$.

We consider the three main noise sources at the TIA stage: the input voltage noise $e_{n}$ of the AN8608 amplifier itself, its input current noise $i_{n}$ and the noise intrinsic to the resistive components of the circuit. These TIA input noise sources are additive, and translate at the TIA output by $e_{\mathrm{TIA}_{\text {out }}}$. The contribution of the amplifier's input voltage noise $e_{n}$ translates at the TIA's ouput by $e_{v}$ with:

$$
e_{v}=e_{n} \frac{A_{0}(\omega)}{1+A_{0}(\omega) \beta(\omega)}
$$

The expression of $e_{v}$ reveals a dependency on the transfer function $H_{\mathrm{TIA}}(\omega)$. The input voltage noise $e_{n}$ is characterised by its Noise Power Spectral Density (NPSD) and is modulated by the noise gain $N_{f}$ of the TIA. $N_{f}$ 's magnitude profile is given in figure 5. For frequencies ranging from DC to the $f_{i+f}$ cutoff $N_{f}=1+R_{f} / R_{\text {SiNw }}$. At $f_{i+f}$, the noise transfer function reaches its first pole. Voltage noise components beyond $f_{i+f}$ will leak though the input capacitor network $C_{i}$ and $N_{f}$ is increasing at $6 \mathrm{~dB}$ octave ${ }^{-1}$. At $f_{f}$ the noise gain is stabilized by the zero formed by the $R_{f} C_{f}$ feedback network of the TIA. Until reaching the second zero in $H_{\text {TIA }}(\omega)$, we have $N_{f}=1+C_{i} / C_{f}$. Finally, beyond the cutoff frequency $f_{\text {roll-off }}=\mathrm{GBP} .1 /\left(1+C_{i} / C_{f}\right)$ the noise gain starts to roll-off at $-6 \mathrm{~dB}$ octave $^{-1}$ [31]. We have:

$$
\left\{\begin{array}{l}
f_{i+f}=\frac{1}{2 \pi R_{f}\left(C_{i}+C_{f}\right)} \simeq 12.63 \mathrm{~Hz} \\
f_{f}=\frac{1}{2 \pi R_{f} C_{f}} \simeq 106 \mathrm{~Hz} \\
f_{\text {roll-off }}=\mathrm{GBP} \cdot \frac{C_{f}}{2 \pi\left(C_{f}+C_{i}\right)} \simeq 1.3 \times 10^{6} \mathrm{~Hz}
\end{array}\right.
$$

The NPSD profile of the amplifier is usually recovered from experimental measurements. The NPSD of the AN8608 amplifier is provided by the manufacturer and given in appendix A. It reveals a $1 / f$ dependency characteristic of many active electronic components at low frequencies. We assume a cutoff frequency $f_{\mathrm{nc}}$ below which the voltage NPSD is inversely proportional to the frequency under consideration. For harmonics superior to $f_{\mathrm{nc}}$ we consider the nominal average NPSD provided by the manufacturer for high-frequencies i.e. $e_{\text {high-freq }}=8 \mathrm{nV} / \sqrt{\mathrm{H}} \mathrm{z}$. The Root Mean Square (RMS) output voltage noise $e_{v}$ can thus be computed as advocated in [32]. The details of this derivation are provided in appendix B. We obtain:

$$
e_{v} \simeq 8.905 \times 10^{-6} \mathrm{~V}_{\mathrm{rms}}
$$

The second input noise source we must consider for the TIA stage is the amplifier's current noise $i_{n}$, which will translate at the TIA's output by the RMS noise potential $e_{i}$ so that:

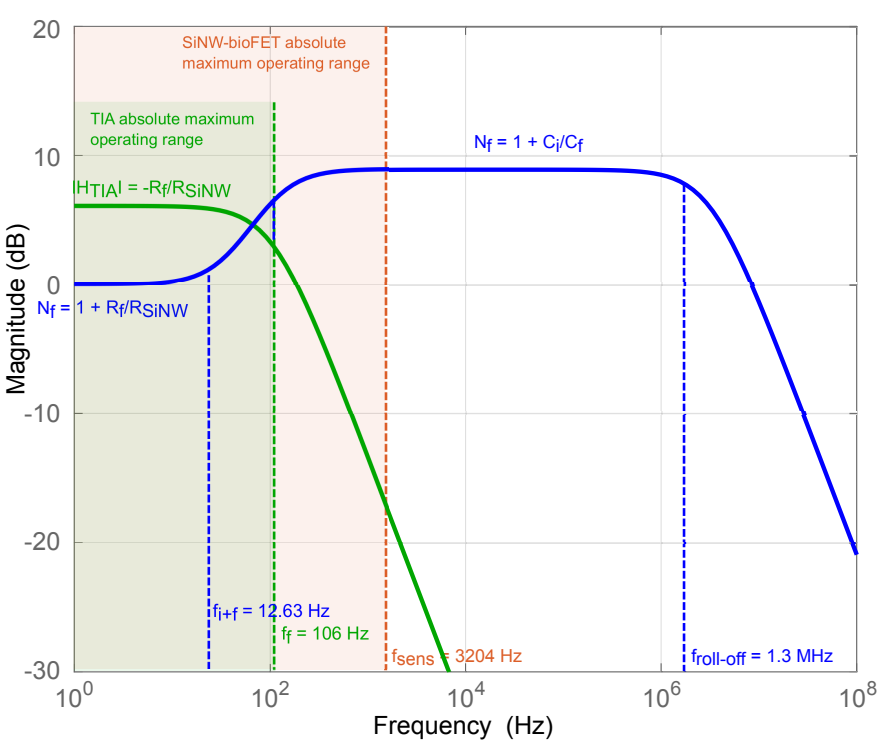

Fig. 5. Closed-loop signal gain magnitude and noise gain Bode plot. The signal gain displays a simple single-pole low-pass filter behavior. Past $f_{f}=$ $1 / 2 \pi R_{f} C_{f}$ the signal gain rolls-off at $-6 \mathrm{~dB}$ /octave. The noise gain instead shows a complex profile as a result of the relations between the amplifier, sensor, shunt capacitance and amplifier feedback network.

$$
e_{i}=\sqrt{\operatorname{ENBW}\left(i_{n} R_{f}+i_{n} R_{\mathrm{b}} N_{f}\right)}
$$

, where ENBW is the Equivalent Noise Bandwidth of the TIA for this configuration. The ENBW is defined so that the RMS noise resulting from a constant NPSD over the ENBW frequency band equals the RMS noise level of the system under consideration, taking into account its noise and gain profiles.

Once again the specification of the current-noise output $e_{i}$ depends on the expression of the noise gain $N_{f}$. The current NPSD is considered constant across the amplifier's bandwidth and according to the AN8608 manufacturer we have $i_{n}=1 \times 10^{-14} \mathrm{~A} / \sqrt{\mathrm{H}} z$. Following a similar reasoning as the one we used for the calculation of $e_{v}$ we can compute the piecewise contribution of the amplifier's current noise throughout the noise gain profile. The details of these calculations are provided in appendix C. We obtain:

$$
e_{i} \simeq 1.3744 \times 10^{-3} \mathrm{~V}_{\mathrm{rms}}
$$

Finally, we must account for the thermal noise generated by the resistive components of the TIA circuitry. Thermal noise is conventionally considered uniform over the frequency spectrum. The noise induced by $R_{f}$ will directly affect $V_{\mathrm{TIA}_{\text {out }}}$ so that:

$$
\begin{gathered}
e_{R_{f}}=\sqrt{4 k_{B} T R_{f} \mathrm{ENBW}} \\
e_{R_{f}}=\sqrt{4 k_{B} T R_{f} \frac{\pi}{2} f_{f}}=2.715 \times 10^{-5} \mathrm{~V}
\end{gathered}
$$

The SiNW-bioFET sensor itself will inject noise at the input of the amplifier. Several studies have demonstrated that the profile of this noise may include a $1 / f$ component [33], 
[34]. As the results supporting these investigations are solely experimental, we assume that the sensor's thermal noise is purely uniform so that:

$$
\begin{gathered}
e_{R_{\text {sens }}}=\sqrt{4 k_{B} T R_{\text {sens }}\left(\frac{R_{f}}{R_{\text {sens }}}\right)^{2} \mathrm{ENBW}} \\
e_{R_{\text {sens }}}=\sqrt{4 k_{B} T R_{\text {sens }}\left(\frac{R_{f}}{R_{\text {sens }}}\right)^{2} \frac{\pi}{2} f_{f}} \\
e_{R_{\text {sens }}}=3.853 \times 10^{-5} \mathrm{~V}
\end{gathered}
$$

The thermal noise originating from $R_{b}$ will be amplified by the noise gain $N_{f}$ (appendix B) and similarly to how we computed $e_{i}$ 's contribution at the TIA output we obtain:

$$
e_{R_{b}} \simeq 2.02 \times 10^{-9} \mathrm{~V}
$$

The contributions of $R_{f}, R_{\text {sens }}$ and $R_{b}$ result in the total RMS thermal noise $e_{R}=4.714 \times 10^{-5} \mathrm{~V}_{\mathrm{rms}}$. Since the voltage, current and thermal noise sources at the TIA output can be assumed uncorrelated, we can add their respective contributions and derive an expression of $e_{\mathrm{TIA}_{\text {out }}}$ :

$$
\begin{aligned}
& e_{\mathrm{TIA}_{\text {out }}}=\sqrt{e_{v}^{2}+e_{i}^{2}+e_{R}^{2}} \\
& e_{\mathrm{TIA}_{\text {out }}}=1.378 \times 10^{-3} \mathrm{~V}_{\mathrm{rms}}
\end{aligned}
$$

Although other current amplification schemes could be envisaged, our analog front-end is limited to this sole singlestage TIA amplifier. This configuration is advantageous in terms of signal-to-noise ratio since an increase in gain in a single-stage design will translate in a proportional increase in signal amplitude, whereas thermal noise, and current-noise will only scale as a function of the square-root of the gain. A single-stage high-gain design is thus preferable over a dualstage design achieving the same signal amplification since, in the latter case, noise at the output of the first stage would scale proportionally with the gain at stage 2 , translating in a poorer overall Signal-to-Noise Ration (SNR) [31].

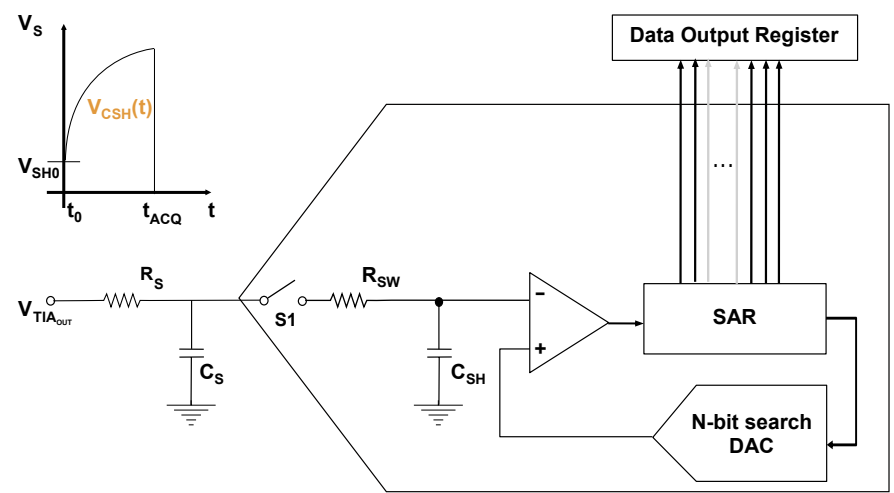

Fig. 6. SAR ADC and RC driver circuit. The $R_{\mathrm{S}} C_{\mathrm{S}}$ network acts both as an anti-alias filter, cutting-off high-frequency noise to input the ADC and as a driving element preventing spurious potential bursts to deteriorate the AD conversion when the switch $S 1$ is closed.

2) $R C$ driver circuit: The next source of noise on the signal conditioning pathway is the $\mathrm{RC}$ filter driving the Analog to
Digital Converter (ADC), entry point to our digital PSD (figure 6). As we saw in the previous section, the high-impedance AC measurements performed on our SiNW-bioFET are limited in bandwidth by the feedback network of the TIA. This sets relatively soft requirements on signal sampling rates. With the aforementioned specifications, the TIA cut-off corresponds to $f_{f}=106 \mathrm{~Hz}$. Such a low-bandwidth application can easily be satisfied using a Successive Approximation Register (SAR) ADC. The external driving network $R_{\mathrm{S}} C_{\mathrm{S}}$ has a dual purpose. First the capacitor $C_{\mathrm{S}}$ is needed in order to prevent spurious bursts or "kicks" to corrupt the input signal and maintain the signal potential throughout the sampling acquisition and conversion time. Second, $R_{\mathrm{S}}$ should help ensure the stability of the TIA or buffer amplifier preceding the ADC stage. The $R_{\mathrm{S}} C_{\mathrm{S}}$ network forms a single pole anti-aliasing filter, that will attenuate high-frequency noise beyond $f_{\text {anti-alias }}=1 / 2 \pi R_{\mathrm{S}} C_{\mathrm{S}}$ $\mathrm{Hz}$.

The $R_{\mathrm{S}} C_{\mathrm{S}}$ driver circuit will thus add thermal noise $e_{R_{\mathrm{S}}}$ to the $V_{\text {TIA }_{\text {out }}}$ signal so that:

$$
e_{R_{\mathrm{S}}}=\sqrt{4 k_{B} T R_{\mathrm{S}} \mathrm{ENBW}}=\sqrt{4 k_{B} T R_{\mathrm{S}} \frac{\pi}{2} f_{\text {anti-alias }}}
$$

The $R_{\mathrm{S}}$ and $C_{\mathrm{S}}$ values should be specified in order to allow sampling speeds that will satisfy the Nyquist-Shannon criteria i.e. $f_{\text {anti-alias }}>2 f_{s_{\text {max }}}$. By setting $R_{\mathrm{S}}=1 \times 10^{3} \Omega$ and $C_{\mathrm{S}}=1 \times 10^{-9} \mathrm{~F}$ the time constant of the $\mathrm{RC}$ filter becomes $1 \times 10^{-6} \mathrm{~s}$. The resolution of the ADC will set the minimum number of time constants for which the S1 switch must remain closed in between acquisitions, so that the potential across $C_{\mathrm{S}}$ can approach that of the input signal with a maximum deviation of a half Less Significant Bit (LSB). For a 12-bit ADC, 9 times constants are sufficient. The best approximation allowed by the SAR ADC could then be reached in $9 \times R_{\mathrm{S}} C_{\mathrm{S}}=9 \mu \mathrm{s}$, limiting the signal bandwidth for the anti-alias filter to $1 / 2 \times 9 / 2 \pi R_{\mathrm{S}} C_{\mathrm{S}} \simeq 55.56 \mathrm{kHz}$, way beyond the bandwidth allowed by our TIA stage (i.e. $106 \mathrm{~Hz}$ ), and thus not impacting the overall bandwidth of the signal conditioning block. With these specifications, the noise added by the $R_{\mathrm{S}} C_{\mathrm{S}}$ network is:

$$
e_{R_{\mathrm{S}}}=\sqrt{4 k_{B} T 10^{3}\left(\frac{\pi}{2} \frac{10^{6}}{2 \pi}\right)}=1.014 \times 10^{-3} \mathrm{~V}
$$

3) Analog to Digital Conversion: Quantization noise is an inevitable consequence of the discretization of the continuous input voltage signal. It is determined by the resolution of the ADC and by the voltage span over which the conversion occurs. For a single-supply 12-bit ADC referenced at $3.3 \mathrm{~V}$, the expression of the quantization noise $e_{q}$ can be shown to be :

$$
e_{q}=\frac{\mathrm{LSB}}{\sqrt{12}}=\frac{3.3 / 2^{12}}{\sqrt{12}}=2.326 \times 10^{-4} \mathrm{~V}
$$

This noise model relies on the assumption that the quantization noise is uncorrelated with the input signal: its power spectral density then spreads about uniformly over the Nyquist 
bandwidth $f_{s} / 2, f_{s}$ denoting the sampling frequency [35]. A non-ideal ADC will inevitably add other spurious noise harmonics to the quantization noise. We disregard the impact of ADC non-idealities as these are not always specified by device manufacturers.

4) Summary: All the aforementioned noise components sum at the output of the signal conditioning block and contribute to the quantized noisy signal $V_{\mathrm{ADC}_{\text {out }}}=V_{\mathrm{PSD}_{\text {in }}}$. The unfiltered digital input signal at the input of the PSD block recovered together with the baseline current for our SiNW-bioFET biased with $V_{\mathrm{DS}}=0.3 \mathrm{~V}$ therefore includes at minima an equivalent RMS noise of amplitude $e_{\mathrm{PSD}_{\text {in }}}=$ $\sqrt{\left(e_{\text {TIA }_{\text {out }}}\right)^{2}+\left(e_{R_{\mathrm{S}}}\right)^{2}+\left(e_{q}\right)^{2}}=1.727 \times 10^{-3} \mathrm{~V}_{\text {rms }}$.

\section{Phase-Sensitive Detection}

Phase-Sensitive Detection (PSD), also sometimes referred to as lock-in amplification, is a technique used in many engineering and scientific disciplines as a solution to varied AC-signals measurement problems. It offers both the possibility to recover signals buried in high levels of noise and to quantify relatively clean signals that may vary in amplitude or frequency over several orders of magnitude [36]. PSD has been leveraged by many for recovering the low current variations of highlyresistive SiNW-bioFETs, outputting signals possibly drown in instrumentation and extrinsic noise. The simplified schematic of a lock-in amplifier is provided in figure 7.

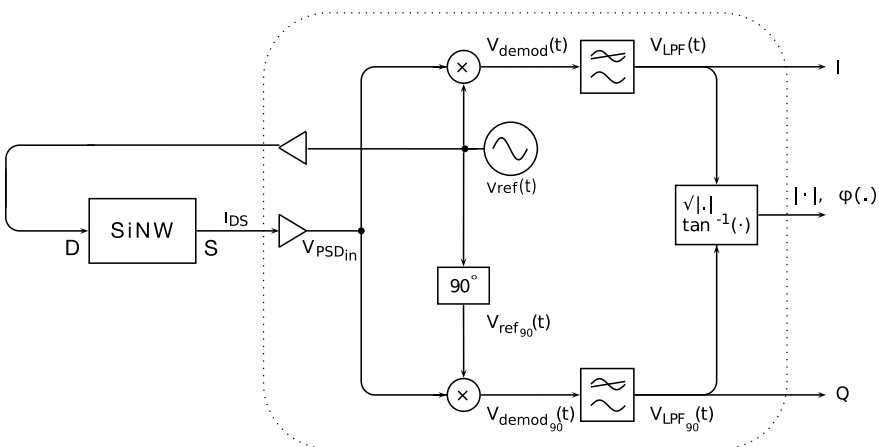

Fig. 7. The lock-in amplifier. Reproduced from [37]

The technique consists in retrieving the magnitude and phase of a periodic AC signal with a known main harmonic pulsation $\omega_{\text {ref }}$. An internal reference signal can be used to generate the excitation signal $V_{\text {ref }}(t)$ applied between the SiNW-bioFET source and drain terminals. $V_{\text {ref }}(t)$ oscillates at a pulsation $\omega_{\text {ref }}$ so that:

$$
V_{\text {ref }}(t)=A_{\text {ref }} \sin \left(\omega_{\text {ref }} t+\phi_{\text {ref }}\right)
$$

The buffered reference signal $V_{\text {ref }}(t)$ can be applied either to the drain or source terminal of the sensor while the other terminal is connected to a fixed reference potential $V_{\text {bias }}$. Let us consider this single-ended configuration: the source potential is maintained at $V_{\text {bias }}=1.65 \mathrm{~V}$ while the reference signal is biased with baseline DC potential of $1.65 \mathrm{~V}$ and oscillates with an amplitude $A_{\text {ref }}$. The amplitude of the excitation signal applied across the SiNW-bioFET is therefore given by $\left|V_{\mathrm{DS}}\right|$, with:

$$
\left|V_{\mathrm{DS}}\right|=\left|V_{\mathrm{D}}-V_{\mathrm{S}}\right|=A_{\text {ref }}
$$

We exclusively consider the cases for which the SiNWbioFET transistor operates linearly, that is that we can assume proportionality between the applied excitation voltage amplitude $V_{\mathrm{DS}}$ and the drain-source current amplitude $I_{\mathrm{DS}}$. Following the signal conditioning block to which we referred to extensively in the previous section, PSD requires the demodulation of the input signal $V_{\mathrm{PSD}_{\text {in }}}(t)$, which corresponds to the digital output signal of the signal conditioning block. In a noise-free scenario, the expression of $V_{\mathrm{PSD}_{\text {in }}}(t)$ depends on the overall transfer function of SiNW-bioFET and of the signal conditioning block. We can write:

$$
V_{\mathrm{PSD}_{\text {in }}}(t)=A_{\mathrm{PSD}_{\text {in }}} \sin \left(\omega_{\text {ref }} t+\phi_{\mathrm{PSD}_{\text {in }}}\right)
$$

1) Demodulation: The first stage of the PSD functional block often consists of an AC-coupling block, in charge of removing the DC baseline offset of the AC signal of interest. PSD then requires the parallel dual-demodulation of the input signal $V_{\mathrm{PSD}_{\text {in }}}(t)$ with the in-line reference signal $V_{\text {ref }}(t)$ and with a second reference signal $V_{\text {ref }_{90}}(t)$ in quadrature with the first, i.e. $\phi_{\text {ref }_{90}}=\phi_{\text {ref }}+90^{\circ}$ (figure 7). In a noise-free scenario, the output of the in-line demodulator can be derived as follows:

$$
\begin{aligned}
V_{\text {demod }}(t)= & V_{\mathrm{PSD}_{\text {in }}}(t) \cdot V_{\text {ref }}(t) \\
V_{\text {demod }}(t)= & A_{\mathrm{PSD}_{\text {in }}} \sin \left(\omega_{\text {ref }} \cdot t+\phi_{\mathrm{PSD}_{\text {in }}}\right) \\
& \times A_{\text {ref }} \sin \left(\omega_{\text {ref }} t+\phi_{\text {ref }}\right) \\
V_{\text {demod }}(t)= & \frac{A_{\mathrm{PSD}_{\text {in }}} A_{\text {ref }}}{2}\left(\cos \left(\phi_{\mathrm{PSD}_{\text {in }}}-\phi_{\text {ref }}\right)\right. \\
& \left.-\cos \left(2 \omega_{\text {ref }} t+\phi_{\text {ref }}+\phi_{\mathrm{PSD}_{\text {in }}}\right)\right)
\end{aligned}
$$

A similar computation for the demodulation with the quadrature reference signal gives:

$$
\begin{aligned}
V_{\mathrm{demod}_{90}}(t)= & V_{\mathrm{PSD}_{\text {in }}}(t) \cdot V_{\text {ref }_{90}}(t) \\
V_{\text {demod }_{90}}(t)= & A_{\mathrm{PSD}_{\text {in }}} \sin \left(\omega_{\text {ref }} \cdot t+\phi_{\mathrm{PSD}_{\text {in }}}\right) \\
& \times A_{\text {ref }_{90}} \sin \left(\omega_{\text {ref }} t+\phi_{\text {ref }}+90\right) \\
V_{\text {demod }_{90}}(t)= & \frac{A_{\mathrm{PSD}_{\text {in }}} A_{\text {ref }_{90}}}{2}\left(\sin \left(\phi_{\mathrm{PSD}_{\text {in }}}-\phi_{\text {ref }}\right)\right. \\
& \left.+\sin \left(2 \omega_{\text {ref }} t+\phi_{\text {ref }}+\phi_{\mathrm{PSD}_{\text {in }}}\right)\right)
\end{aligned}
$$

Both the expressions of the in-line and quadrature demodulators output let appear a DC component and a harmonic term pulsating at twice the excitation frequency. The DC components hold information about both the amplitude and phase of the PSD input signal $V_{\mathrm{PSD}_{\text {in }}}$, which will allow us to recover information on $I_{\mathrm{DS}}$ later on. The purpose of this dual-demodulation process is to bring the $V_{\mathrm{PSD}_{\text {in }}}(t)$ amplitude and phase information to DC. The key to the PSD process resides in the subsequent low-pass filtering of both in-line and quadrature demodulated signals. If we consider an ideal filter with a cut-off pulsation $\omega<\omega_{\text {ref }}$ we would expect at the filters output: 


$$
\left\{\begin{array}{l}
V_{\mathrm{LPF}}(t)=\frac{A_{\mathrm{PSD}_{\text {in }}} A_{\mathrm{ref}}}{2} \cos \left(\phi_{\mathrm{PSD}_{\mathrm{in}}}-\phi_{\mathrm{ref}}\right) \\
V_{\mathrm{LPF}_{90}}(t)=\frac{A_{\mathrm{PSD}_{\text {in }}} A_{\mathrm{ref}_{90}}}{2} \sin \left(\phi_{\mathrm{PSD}_{\text {in }}}-\phi_{\mathrm{ref}}\right)
\end{array}\right.
$$

We define $A_{\text {ref }}=1 \mathrm{~V}$ and $\phi_{\text {ref }}=0$. We eventually obtain:

$$
\left\{\begin{array}{l}
V_{\mathrm{LPF}}(t)^{2}+V_{\mathrm{LPF}_{90}}(t)^{2}=\frac{\left(A_{\mathrm{PSD}_{\text {in }}}\right)^{2}}{4} \\
\frac{V_{\mathrm{LPF}_{90}}(t)}{V_{\mathrm{LPF}}(t)}=\tan \left(\phi_{\mathrm{PSD}_{\text {in }}}\right)
\end{array}\right.
$$

We can now recover the amplitude and phase of $V_{\mathrm{PSD}_{\text {in }}}(t)$ as:

$$
\left\{\begin{array}{l}
\left|V_{\mathrm{PSD}_{\text {in }}}\right|=A_{\mathrm{PSD}_{\text {in }}}=2 \sqrt{V_{\mathrm{LPF}}(t)^{2}+V_{\mathrm{LPF}_{90}}(t)^{2}} \\
\angle V_{\mathrm{PSD}_{\text {in }}}=\phi_{\mathrm{PSD}_{\text {in }}}=\arctan \left(\frac{V_{\mathrm{LPF}_{90}(t)}\left(V_{\mathrm{LPF}}(t)\right.}{}\right)
\end{array}\right.
$$

The $I_{\mathrm{DS}}$ current phase is accordingly given by $\angle I_{\mathrm{DS}}=\phi_{\mathrm{PSD}_{\text {in }}}$ and its magnitude $\left|I_{\mathrm{DS}}\right|$ is function of the various gains and attenuations in effect throughout the signal conditioning block. In the non-ideal case, the input signal $V_{\mathrm{PSD}_{\text {in }}}(t)$ will be corrupted by spurious signal components sourced by intrinsic noise, extrinsic noise, or resulting from low-frequency signal drift or DC errors. If $V_{\mathrm{PSD}_{\text {in }}}(t)$ incorporates a DC component, then the in-line demodulation of $V_{\mathrm{PSD}_{\text {in }}}(t)$ gives:

$$
\begin{aligned}
V_{\text {demod }}(t)= & V_{\mathrm{PSD}_{\text {in }}}(t) \cdot V_{\omega_{\text {ref }}}(t) \\
V_{\text {demod }}(t)= & \left(A_{\mathrm{PSD}_{\text {in }}} \sin \left(\omega_{\text {ref }} \cdot t+\phi_{\mathrm{PSD}_{\text {in }}}\right)+C\right) \\
& \times A_{\text {ref }} \sin \left(\omega_{\text {ref }} t+\phi_{\text {ref }}\right) \\
V_{\text {demod }}(t)= & \frac{A_{\mathrm{PSD}_{\text {in }}} A_{\text {ref }}}{2}\left(\cos \left(\phi_{\mathrm{PSD}_{\text {in }}}-\phi_{\text {ref }}\right)\right. \\
& \left.-\cos \left(2 \omega_{\text {ref }} t+\phi_{\text {ref }}+\phi_{P S D_{\text {in }}}\right)\right) \\
& +C A_{\text {ref }} \sin \left(\omega_{\text {ref }} t+\phi_{\text {ref }}\right)
\end{aligned}
$$

The DC offset input has shifted to the pulsation $\omega_{\text {ref }}$. This last equation illustrates the importance of the AC-coupling block for cancelling of the input signal DC component before it generates a spurious harmonic of large amplitude at the filter input.

The precision of the PSD signal recovery process thus depends on the level of noise corrupting $V_{\mathrm{PSD}_{\text {in }}}(t)$. Specifically, the demodulation of noise harmonics at frequencies close to $f_{\text {exc }}$ will fall in the transmission window of the filter and remain largely unattenuated. For systems exhibiting purely white noise, the improvement in Signal-to-Noise Ration (SNR) offered by PSD is specified by:

$$
\text { Improvement Factor }=\frac{\mathrm{SNR}_{\text {out }}}{\mathrm{SNR}_{\text {in }}}=\frac{B_{\text {in }}}{B_{\text {out }}},
$$

where $B_{\text {in }}$ is the Equivalent Noise Bandwidth (ENBW) at the input of the PSD block and $B_{\text {out }}$ is the ENBW at its output [36]. In our specific case though, the spectrum of the signal at the output of the signal conditioning block is likely to contain various correlated noise components, including $1 / f$ noise sourced from the amplifier and from the SiNWbioFET itself. As highly-sensitive SiNW-bioFETs will exhibit small dimensions or may be biased in a high-resistive regime, we may not be entitled to choose a measurement excitation frequency beyond the $1 / f$ noise cut-off $f_{\mathrm{nc}}$ and may need to carry out $\mathrm{AC}$ measurements within the $1 / f$ region. The expression of the improvement factor (equation 54) then does not hold anymore and a more thorough investigation of the influence of the PSD stage on the output SNR is needed.

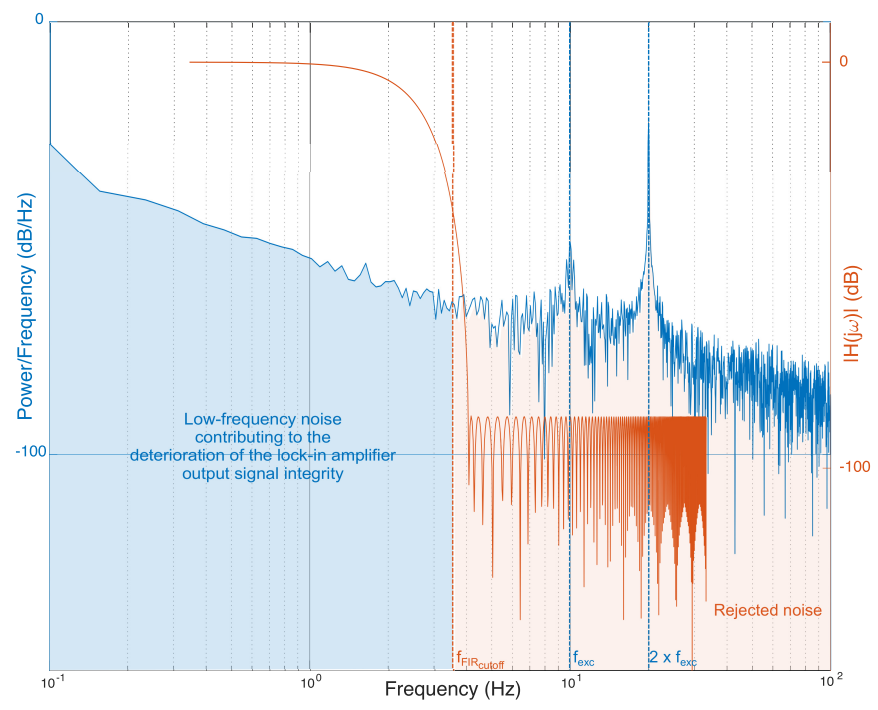

Fig. 8. Magnitude spectrum of a digitized $10 \mathrm{~Hz}$ demodulated signal (in blue) and frequency response of a 120 taps FIR filter (in orange). The filter frequency response illustrates the noise components that will remain mostly unattenuated: those below $f_{\mathrm{FIR}_{\text {cutoff }}}$

We consider a sinusoidal excitation signal at $f_{\text {exc }}=10 \mathrm{~Hz}$. At such a low frequency, the transamplification of $I_{\mathrm{DS}}$ is corrupted by the amplifier $1 / f$ noise, rolling off at $-3 \mathrm{~dB} /$ octave. Figure 8 illustrates the magnitude spectrum of the demodulated signal. The energy of the input signal initially oscillating at $10 \mathrm{~Hz}$ is split, and for half brought back to DC (that is the signal component we wish to recover) and for half transposed at $20 \mathrm{~Hz}$, twice the excitation frequency. Here, a $10 \mathrm{~Hz}$ pulsation remains as a result of the non-ideal AC-coupling of the signal at the demodulator's input. Figure 8 also illustrates the challenge of rejecting noise when the excitation frequency is low. $1 / f$ noise, the projection of DC offsets to $f_{\text {exc }}$ and the projection of half of the energy of the main harmonic signal at $2 \times f_{\text {exc }}$ will sum as they occupy a narrow frequency spectrum close to DC. Therefore, the lower the excitation frequency, the more stringent the requirements on the low-pass filtering stage. We now elaborate on the implications of these aspects on the filter design requirements.

2) Low-Pass FIR Filtering: The continuous lock-in recovery of SINW-bioFET current signals is governed by an intrinsic trade-off: high TIA gains and narrow operation bandwidth are likely to corrupt signal integrity by translating in significant amount of thermal and current noise, thus necessitating lowcutoff, and sharp roll-off filters. High-order filters, meeting 
drastic stop-band attenuation and sharp transition band requirements will, in turn, exhibit longer time constants limiting the throughput of measurement points. As we only consider the recovery of steady-state $I_{\mathrm{DS}}$ currents, we assume that prolonged acquisition times are acceptable and thus that we may rely on sharp digital filters presenting large number of taps. We harnessed the Parks-McClellan algorithm to design six FIR filters of respectively 17, 45, 72, 120, 359 and 449 taps, respecting tolerances of $0.01 \mathrm{~dB}$ of ripple in the passband and a large $60 \mathrm{~dB}$ of ripple allowed in the stopband (appendix F). These filters enable attenuations superior to $-90 \mathrm{~dB}$ in the stopband.

Just like we computed the RMS noise level at the TIA output, we can deduce from the NPSD at the output of the demodulator what is the RMS noise level at the output of the filter. We may observe that the demodulation process shifted the NPSD frequency spectrum at the input of the demodulator (i.e. the NPSD at the output of the signal conditioning block) to the left of the frequency domain by an amount of $f_{\text {exc }} \mathrm{Hz}$. As illustrated in figure 8 , the total RMS noise $e_{\mathrm{PSD}_{\text {out }}}$ at the PSD block output will result from the contributions of the noise harmonics (blue line) laying within the FIR filter passband (orange line).

A piecewise calculation of these contributions can be derived similarly to what was presented for the various TIA noise contributions. If we assume the ideal subtraction of the signal DC offset before demodulation, the calculation for $e_{\mathrm{PSD}_{\text {out }}}$ becomes:

$$
\begin{aligned}
e_{\mathrm{PSD}_{\mathrm{out}}}= & \sqrt{e_{v_{\text {-ENBW }}}^{2}+e_{i_{\mathrm{ENBW}}}^{2}+e_{R_{\mathrm{ENBW}}}^{2}+e_{R_{\mathrm{S}-\mathrm{ENBW}}}^{2}+e_{q_{\text {-ENBW }}}^{2}} \\
& +V_{\left(2 f_{\mathrm{exc}}\right)_{\mathrm{rms}}}
\end{aligned}
$$

, where $e_{v_{\text {-ENBW }}}, e_{i_{\text {-ENBW }}}, e_{R_{\mathrm{ENBW}}}, e_{R_{\mathrm{S}-\mathrm{ENBW}}}, e_{q \text {-ENBW }}$ the contributions after demodulation and FIR filtering of the amplifier's voltage noise, current noise, of the thermal noise of $R_{b}$ and $R_{f}$, of $R_{\mathrm{S}}$, of the quantization noise and of the spurious harmonic remaining at $2 \times f_{\text {exc }}$ respectively. The details for their respective derivations is provided in appendix E. Importantly, $e_{\mathrm{PSD}_{\text {out }}}$ corresponds to the average RMS noise level we expect to corrupt our lock-in amplified $I_{\mathrm{DS}}$ signal. Finally, we may recover the theoretical Limit Of Detection for our SiNWbioFET/Lock-in amplifier system from $e_{\mathrm{PSD}_{\text {out }}}$, with the LoD defined as:

$$
\text { LoD }=3 \times \text { Noise } \text { floor }_{\mathrm{RMS}}
$$

\section{METHODS}

We implemented a Matlab analytical model of the ATP detection system described in the previous section. We established the relations binding the SiNW-bioFET properties, to those of the biological sample immersing the gate, to the signal conditioning block and to the PSD block. We investigated the influence of various design parameters i.e. the variables under the control of the various system engineers, on the overall SiNW-bioFET/lock-in amplifier system sensitivity. Our overall objective was, from this sensitivity analysis, to identify optimal designs and recommend specific system operation configurations. The initial specifications for the SiNW-bioFET, for the biological matrix and for the instrumentation are given in Table I.

\begin{tabular}{|c|c|c|c|}
\hline Sub-system & Component & Specification & Value \\
\hline \multirow[b]{2}{*}{ SiNW-bioFET } & SiNW & $\begin{array}{c}\text { Length } \\
\text { Radius } \\
\text { Carrier mobility } \\
\text { Boron doping } \\
\text { Stray capacitance }\end{array}$ & $\begin{array}{c}20 \times 10^{-6} \mathrm{~m} \\
20 \times 10^{-9} \mathrm{~m} \\
2 \mathrm{~cm}^{2} / \mathrm{Vs} \\
1 \times 10^{24} \mathrm{~m}^{-3} \\
1 \times 10^{-13} \mathrm{~F}\end{array}$ \\
\hline & Gate & $\begin{array}{c}\text { Material } \\
\text { Thickness } \\
\text { Receptor } \\
\text { Receptor projection area } \\
\text { Receptor charge } \\
\text { Receptor density } \\
\text { Ligand } \\
\text { Ligand projection area } \\
\text { Ligand charge } \\
\text { Charge-surface distance }\end{array}$ & $\begin{array}{c}\mathrm{AlO}_{2} \\
5 \times 10^{-9} \mathrm{~m} \\
\mathrm{ABL} \text { Tyrosine-kinase } \\
3 \times 10^{-17} \mathrm{~m}^{2} \\
- \\
65 \times 10^{12} \text { receptors } / \mathrm{m}^{2} \\
\text { ATP } \\
3 \times 10^{-17} \mathrm{~m}^{2} \\
-3 e \\
2.4 \mathrm{~nm}\end{array}$ \\
\hline Sample & & $\begin{array}{c}\text { Ionic strength } \\
\text { Temperature } \\
\text { ATP concentration }\end{array}$ & $\begin{array}{c}1 \times 10^{-12} \mathrm{~mol} \mathrm{~m}^{-3} \\
298 \mathrm{~K} \\
\text { var }\end{array}$ \\
\hline \multirow{4}{*}{ Signal conditioning circuitry } & Excitation & $\begin{array}{l}\text { Frequency } \\
\text { Amplitude }\end{array}$ & $\begin{array}{c}\text { var } \\
0.3 \mathrm{~V}\end{array}$ \\
\hline & TIA & $\begin{array}{c}\text { Opamp } \\
\text { GBW } \\
C_{\mathrm{cm}} \\
C_{\text {diff }} \\
I_{\text {noise }} \\
e_{v} \\
R_{\mathrm{f}} \\
C_{\mathrm{f}}\end{array}$ & $\begin{array}{c}\text { AN8608 } \\
1 \times 10^{7} \mathrm{~Hz} \\
8 \times 10^{-12} \mathrm{~F} \\
2.2 \times 10^{-12} \mathrm{~F} \\
1 \times 10^{-14} \mathrm{~A} / \sqrt{\mathrm{H} z} \\
8 \times 10^{-9} \mathrm{~V} \sqrt{\mathrm{Hz}} \\
\text { var } \\
1.5 \times 10^{-11} \mathrm{~F}\end{array}$ \\
\hline & RC Driver & $\begin{array}{l}R_{\mathrm{S}} \\
C_{\mathrm{S}} \\
\end{array}$ & $\begin{array}{c}1 \times 10^{3} \Omega \\
1 \times 10^{-9} \mathrm{~F}\end{array}$ \\
\hline & $\mathrm{ADC}$ & $\begin{array}{c}\text { Resolution } \\
\text { Sampling frequency } \\
\text { Input span }\end{array}$ & $\begin{array}{l}12 \text { bits } \\
4 \times f_{s} \\
3.3 \mathrm{~V}\end{array}$ \\
\hline Phase Sensitive Detector & $\begin{array}{l}\text { AC-coupling } \\
\text { Demodulation } \\
\text { FIR Filtering }\end{array}$ & $\begin{array}{l}\text { Output offset error } \\
\text { Frequency } \\
\text { ENBW }\end{array}$ & $\begin{array}{l}\overline{\mathrm{f}_{\mathrm{exc}}} \\
\text { var }\end{array}$ \\
\hline
\end{tabular}

TABLE I

INITIAL SYSTEM SPECIFICATIONS

As we saw in the previous sections, instrumentation properties are likely to play a role in determining the overall LoD (and best resolution) of our SiNW-bioFET/lock-in amplifier assembly. For highly-resistive SiNW channels, e.g. presenting nanoscale cross-sectional areas or biased in the subthreshold regime, the sole intrinsic noise of the signal acquisition chain puts the theoretical high-sensitivity of the biosensor in jeopardy. Since the SiNW length does not play a role in defining SiNW-bioFET sensitivity (at least when length is sufficient to avoid short-channel effects altogether), it presents an opportunity: shorter SiNWs will show a lower intrinsic resistance, requiring lower gains, and enabling larger measurement bandwidth possibly pushing the excitation frequency beyond the $1 / f$ window of the active components in the circuit. SiNW length, excitation frequency, dopant concentration (arguably allowing the simulation of transistor operation in highly depleted regimes) and of course FIR filter design are therefore critical in defining the overall system LoD. We carried out a parametric study investigating the influence of these parameters on the SiNW-bioFET/lock-in amplifier LoD.

As we mentioned before, noise components at the TIA input only scale with the square-root of the gain of the TIA, $-R_{f}$. This gain should therefore be set to the maximum allowed value in order to provide the best overall SNR. As the AN8608 amplifier is operated between ground and $3.3 \mathrm{~V}$, AC signals amplified to a maximum amplitude of $3.3 / 2=$ $1.65 \mathrm{~V}$ were tolerated. The gain specification was decided upon iteratively for each SiNW-bioFET/lock-in amplifier configuration, starting at $R_{f}=1 \mathrm{G} \Omega$, and decreased by one order 
of magnitude if saturation was detected at the TIA output for any of the measurements over the ATP concentration range $[0-1] \mathrm{mol} \mathrm{m}^{-3}$.

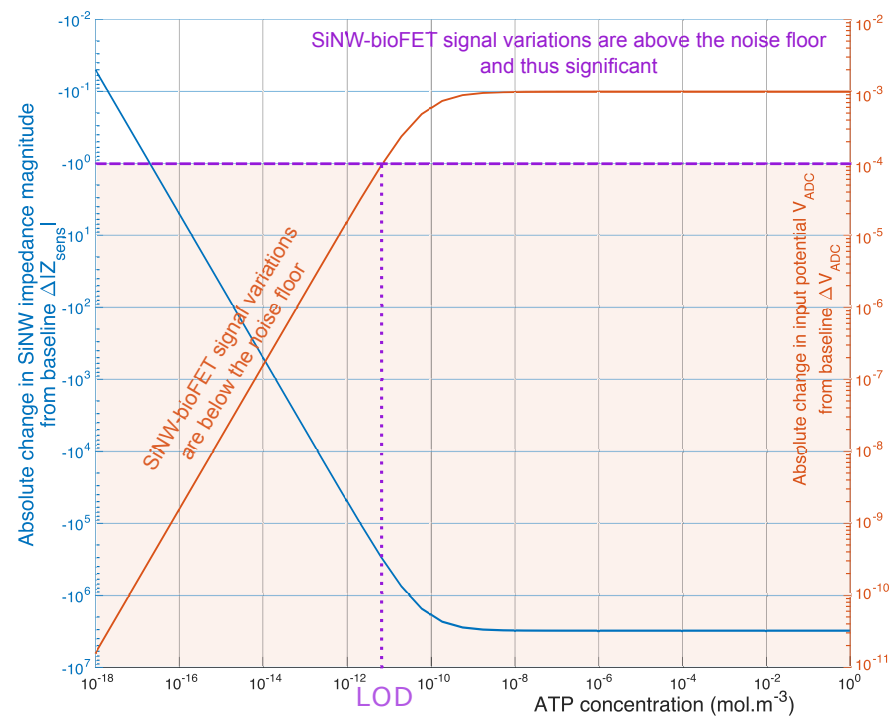

Fig. 9. SiNW-bioFET response to ATP and Limit of Detection. From infinitesimal ATP concentration, spurious noise still appears at the output of the lock-in amplifier. The noise floor, equivalent to $3 \times e_{\mathrm{PSD}_{\text {out }}}$ is represented by the horizontal purple line. Any signal variation observed at the output of the lock-in amplifier below this level should be considered as noise Variations of amplitude superior to this level can be considered significant. We may interpolate the ATP concentration for which the noise floor is reached: this concentration corresponds to the SiNW-bioFET/Lock-in amplifier system LoD.

We made sure that all the measurement points acquired for a sweep of ATP concentration were obtained with an identical TIA gain $-R_{f}$. Indeed if binding of a greater number of target analytes were to necessitate a readjustment of the gain i.e. if the signal were to saturate at the TIA output, then the noise calculation would need to be reiterated. Furthermore, any change in the SiNW resistance cascades in changes in noise levels throughout the acquisition and signal processing chains (e.g. from a shifting in the TIA noise gain profile $N_{f}$ ). We assumed these changes were negligible and carried out our noise calculations for each sweep in ATP concentration using the intrinsic SiNW resistance $R_{\text {SiNW }}$ i.e. at $[\mathrm{ATP}]=$ $0 \mathrm{~mol} \mathrm{~m}^{-3}$. After considering the limits beyond which device fabrication and microfluidics integration becomes challenging, we limited our parametric investigations to SiNWs presenting lengths in the $1-20 \mu \mathrm{m}$ range. Excitation frequency was swept from $f_{\text {exc }}=1 \times 10^{-2} \mathrm{~Hz}$ to three orders of magnitude beyond the TIA bandwidth cutoff of each configuration, or $f_{\max }=\left(1 \times 10^{3}\right) / 2 \pi R_{f} C_{f} \mathrm{~Hz}$.

We were able to recover the LoD for each configuration by relying on the ideal response curves for the SiNW-bioFET such as the one presented in figure 9. By interpolating the analyte concentration for which the corresponding variation in voltage signal change is $3 \times e_{\mathrm{PSD}_{\text {out }}}$ (equation 55) we could retrieve the system-level LoD performance.

\section{RESULTS}

Results for acquisitions using our 17 taps (FIR17) and our 449 taps (FIR449) FIR filters reveal the most important findings of this study. Figure 10 and 11 respectively display the best-case scenario LoDs one may hope to achieve, for a given instrumentation hardware configuration, as SiNW channels vary in length, and as a function of the excitation frequency of the lock-in amplifier.

For a given FIR filter, we observe quite predictably a general trend towards higher sensitivities (e.g lower LoD) as SiNW dopant concentration decreases. Indeed, for dopant concentrations [dopant] $=1 \times 10^{24}, 1 \times 10^{23}, 1 \times 10^{22}$, and $1 \times 10^{21} \mathrm{~m}^{-3}$ the best-case scenario LoD for FIR17 lowers from $2.98 \times 10^{-4}$ to $7.5 \times 10^{-6}, 2.23 \times 10^{-6}$ and $1.77 \times 10^{-6} \mathrm{~mol} \mathrm{~m}^{-3}$ respectively. This general behaviour is expected from the analytical model of the SiNW-bioFET sensor alone. Just as predictably, a comparison of minima in sub-figures 11a to 11d (FIR449) with their counterparts in figure 10 (FIR17) reflects the intuitive benefit of sharper rolloff filters: these help rejecting noise better, which in turn favors lower LoD values. Choosing FIR449 instead of FIR17, thus brings down the best achievable LoD level within this design space from $1.77 \times 10^{-6} \mathrm{~mol} \mathrm{~m}^{-3}$ to $7.21 \times 10^{-7} \mathrm{~mol} \mathrm{~m}^{-3}$, or the equivalent of a 2.45 -fold increase in LoD performance.

More interestingly now, we observe in both figure 10 and figure 11 behaviours we would not expect from an analysis of the sensor alone. First, although the least-doped sensors provide the single highest LoD performance for this design space (sub-figures $10 \mathrm{~d}$ and 11d), LoD performances over most of the design space are actually worse-off at [dopant] $=1 \times 10^{21} \mathrm{~m}^{-3}$, than for sensors doped 10 times more. Indeed, minima in figure 10d and figure 11d are guarded in between the steep walls of the "slanted half-pipe" surface curve that describes LoD performances for the most poorly doped SiNW channels. On the opposite, LoD performance curves for [dopant] $=1 \times 10^{22} \mathrm{~m}^{-3}$ (sub-figures 10c and 11c) are relatively flat. The slanted half-pipes of sub-figures $10 \mathrm{~d}$ and 11d translate the stronger dependency of detection limits on both lock-in excitation frequency and on SiNW length, as the SiNW channel dopant concentration is reduced. This peculiar behaviour is a consequence of the limitations of the lockin amplifier. As the baseline resistivity of the SiNW channel increases, the instrument cannot cope with the drop in baseline signal amplitude. The noise injected downstream of the SiNWbioFET by the signal conditioning electronic components progressively represents a larger part of the signal recovered by the PSD stage of the amplifier. The latter, depending on the performance of its filtering stage, cannot withstand the decrease in SNR of the input signal. Noise eventually leaks through the FIR filter and LoD performances decrease. This decrease in performance is aggravated both at higher and lower excitation frequencies. At higher frequencies, two detrimental effects combine: the sharp increase in the TIA noise gain $N_{f}$ (for $f_{\text {exc }}>f_{i+f}$ ), and the TIA signal gain starting to rolloff as $f_{\text {exc }}$ approaches the TIA $R_{f} C_{f}$ cutoff. This behaviour directly translates the operation range limits we discussed in the introduction: $f_{\text {exc }}<\min \left(f_{f}, f_{i+f}\right)$. At lower frequencies now, we observe the clear impact of $1 / f$ noise on system-level LoD as SiNW channel dopant concentration is reduced. This is especially true for the least performing FIR filter (FIR17), which rejects lower frequency harmonics very poorly when 


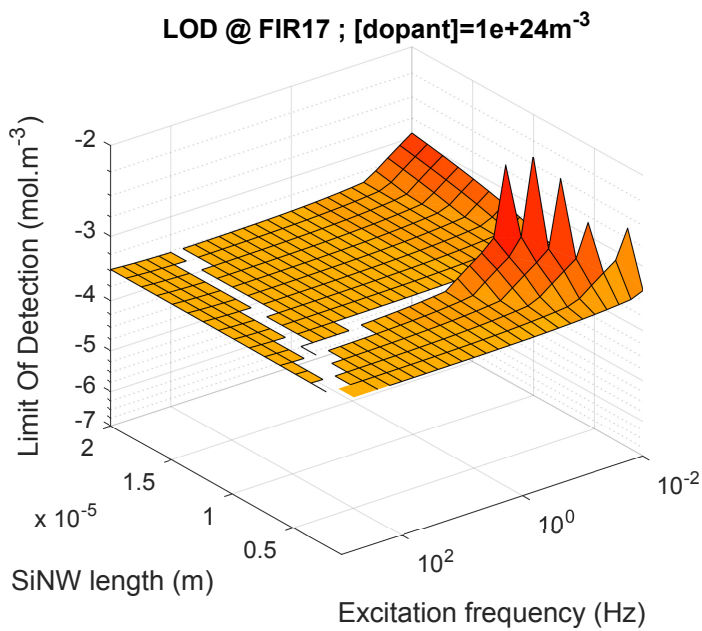

(a)

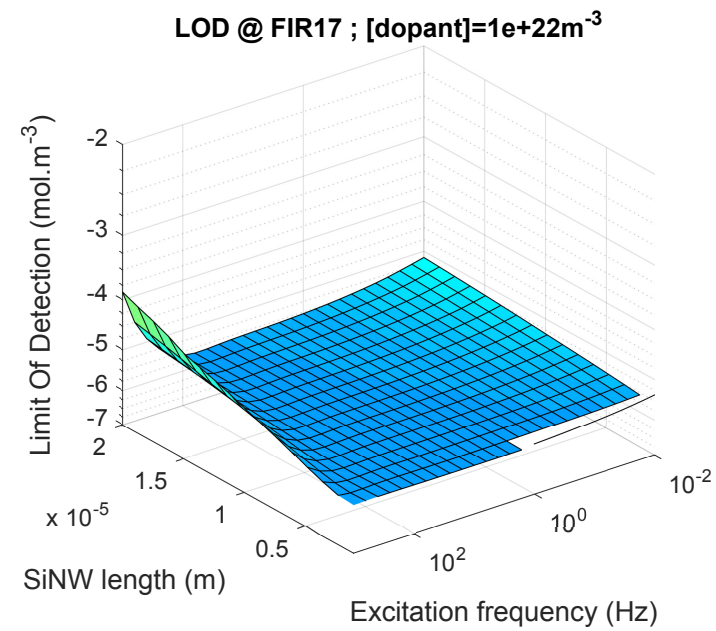

(c)
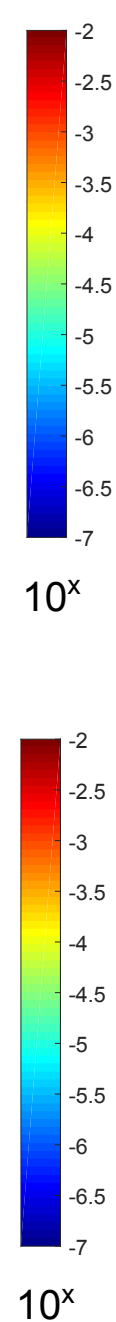

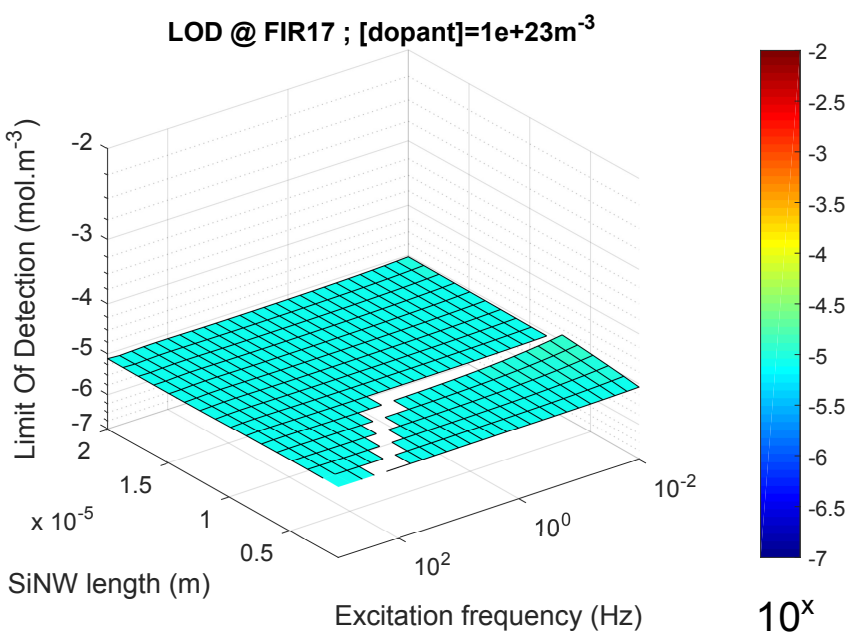

(b)

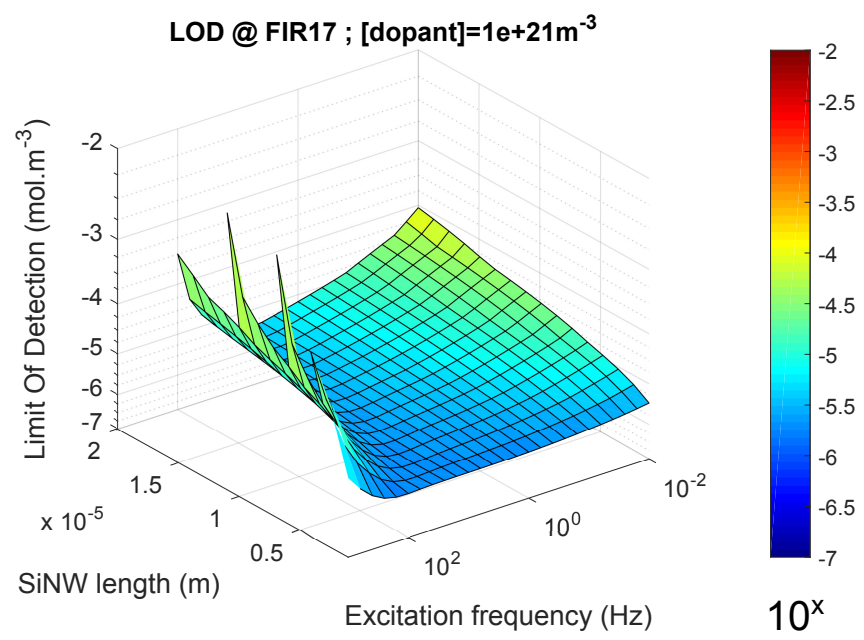

(d)

Fig. 10. a-d - Best-case scenario LoD for PSD with the FIR17 filter for various SiNW dopant concentrations. The best performance is LoD = $1.77 \times 10^{-6} \mathrm{~mol} \mathrm{~m}^{-3}$. It is achieved for a SiNW dopant concentration of $1 \times 10^{21}$ dopant $/ \mathrm{m}^{3}$ for a SiNW length of $1 \mu \mathrm{m}$, at an excitation frequency of $f_{\mathrm{exc}}=100 \mathrm{~Hz}$.

FIR449 performs reasonably well.

Figures $10 \mathrm{~d}$ and $11 \mathrm{~d}$ also reveal stronger dependency of LoD performances on SiNW length. This dependency is explained by the same reason behind the greater dependency on excitation frequency: a greater amount of noise is injected in the acquisition signal if the SiNW is lengthened. We can see that this dependency is not as strong for FIR449 as it is for FIR17, once again a consequence of the sharper roll-off filter being more resilient to noise injection.

Finally, figure 10a reveals another interesting behaviour. The LoD performance surface curve appears to be fragmented in 3 distinct surfaces, and a significant LoD step-response is associated with devices which lengths decrease below the $7 \mu \mathrm{m}$ threshold. Notice also that this step is curled, depicting both increasing $\mathrm{LoD}$ values as excitation frequencies tend toward DC and seemingly a return to lower LoD values as SiNW length is reduced further. This behaviour is the consequence of the availability of a discrete and limited number of gains for the TIA stage. Indeed, the successive gains of the current amplifier are one order of magnitudes apart from one another
$\left(R_{f}=1 \times 10^{9}, 1 \times 10^{8} \Omega \ldots\right)$. For the very short SiNW channels, a lower gain may need to be selected in order to prevent signal saturation. This is what happens at the critical threshold $L=7 \mathrm{~nm}$ : the selection of a lower gain, brings down the baseline signal at the TIA output, meaning that the noise injected downstream of the acquisition chain (i.e. at the RC driver circuit stage and at digitization) will represent a larger portion of the acquired signal. Also note that switching to a smaller feedback resistance $R_{f}$ will decrease the noise it generates following a square-root relationship, whereas the effective signal will decrease proportionally to $R_{f}$. These phenomena will contribute to degrade the SNR at the input of the PSD. This, in turn, inevitably results in higher LoDs. The reason the LoD flattens again as SiNW lengths are reduced is that the relative increase in noise induced by the selection of a smaller TIA gain is compensated by shorter channels being less resistant, and therefore injecting less noise downstream of the acquisition chain. One can notice once again that the system-level consequences of these effects are more pronounced for FIR17 than for FIR449: the latter filter 


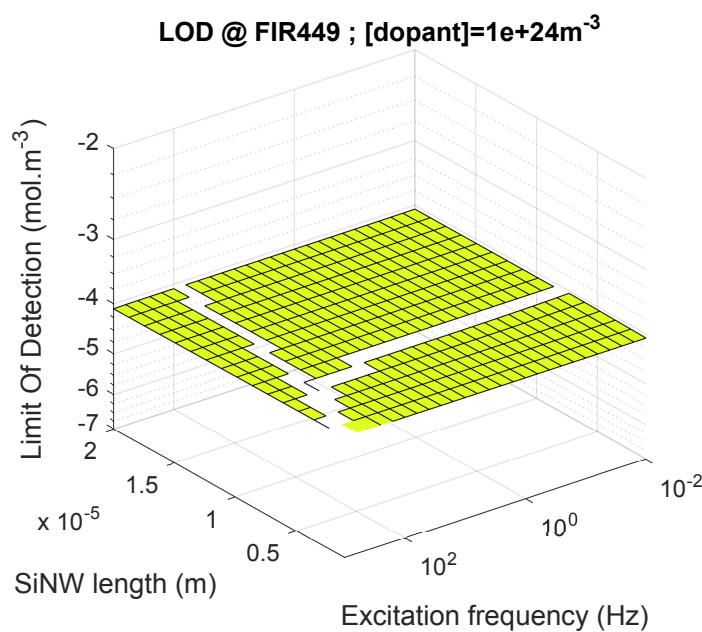

(a)

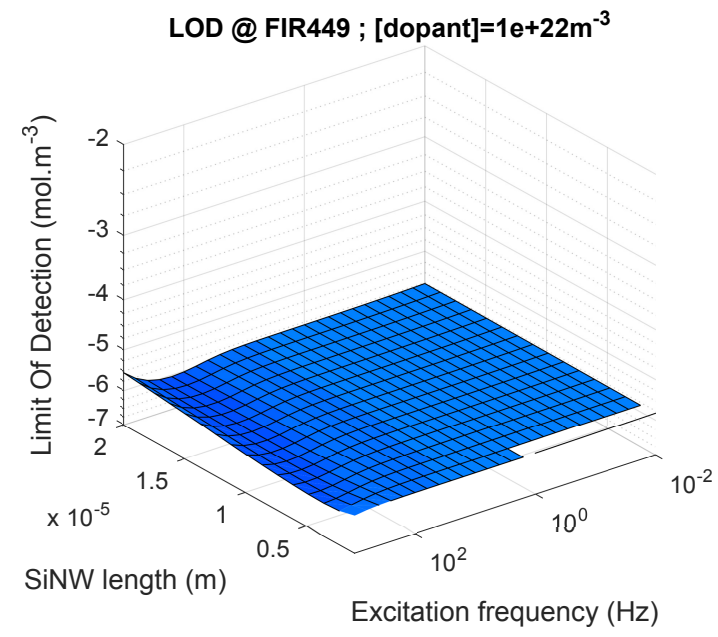

(c)
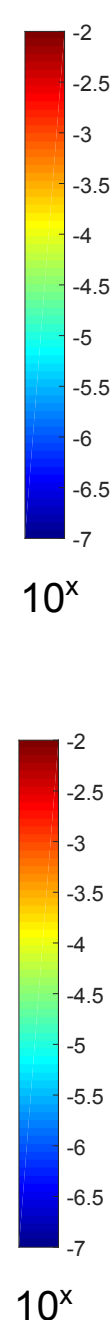

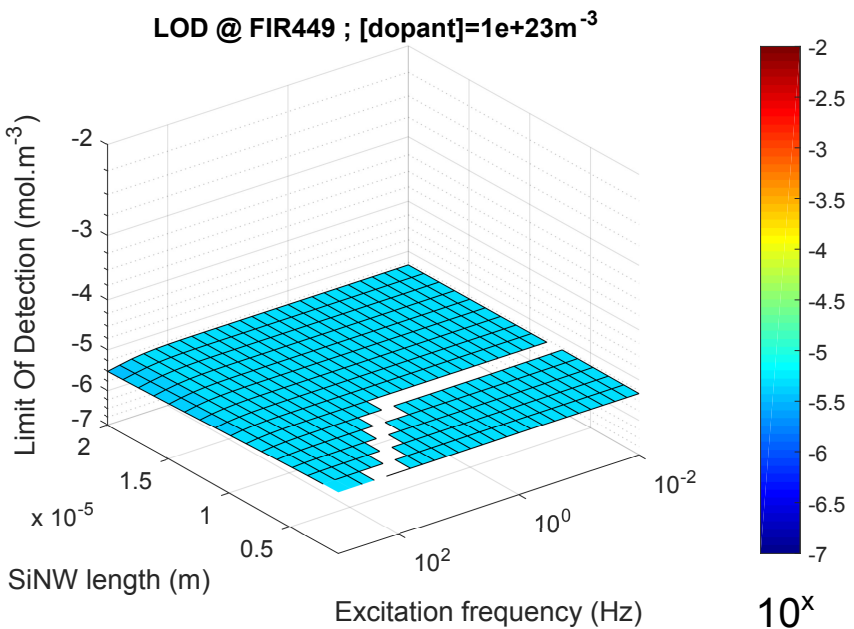

(b)

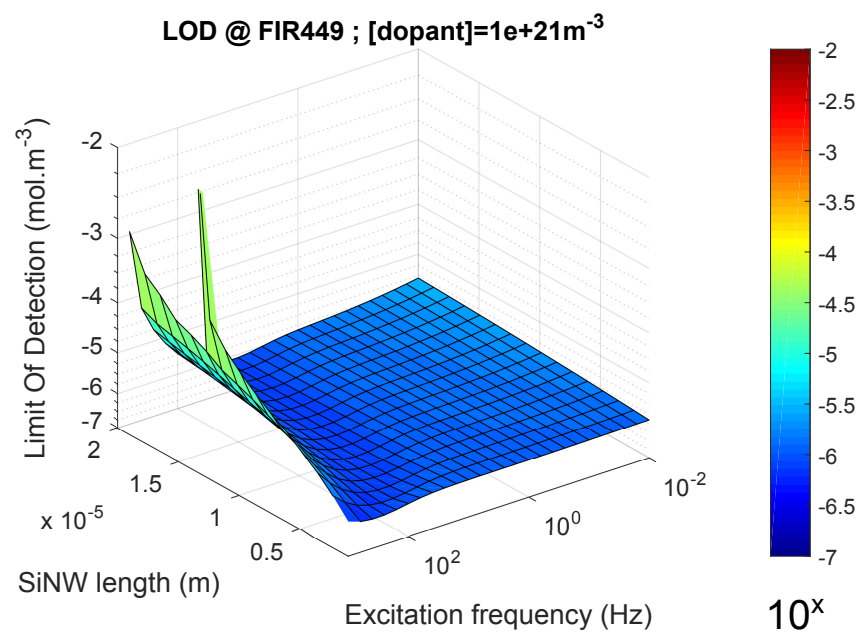

(d)

Fig. 11. a-d - Best-case scenario LoD for PSD with the FIR449 filter for various SiNW dopant concentrations. The best performance is LoD = $7.21 \times 10^{-7} \mathrm{~mol} \mathrm{~m}^{-3}$. It is achieved for a SiNW dopant concentration of $1 \times 10^{21}$ dopant $/ \mathrm{m}^{3}$, at an excitation frequency $f_{\text {exc }}=398 \mathrm{~Hz}$ and for a SiNW length of $1 \mu \mathrm{m}$. The higher noise rejection capabilities of the FIR449 filter need to be traded off against longer filter settling times.

benefits from a lower cut-off frequency and from a sharper roll-off which allow it to be more resilient to a decrease in SNR.

\section{Discussion}

Much effort has been spent on the development of novel SiNW-bioFET architectures and on the investigation of their behaviours. Our understanding of underlying science, together with advances in SiNW-bioFET design and fabrication techniques have progressively driven down SiNW-bioFET LoD levels, mostly via the successfull trade-off of a set of now well-known sensor design parameters: SiNW geometry, carrier dopant concentration, operation regime, gate oxide material and thickness, and the physico-chemical properties of the biofunctionalization layer. Although various designs have demonstrated sub-femtomolar detection capabilities, our investigation shows that the maximization of SiNW-bioFET design performances alone cannot always permit the maximisation of performances of the sensor-instrumentation integration. A focus on systemic performances should yet prevail if SiNWbioFET technology is to be more easily translated into diagnostic practices.

Our research addressed this limitation by demonstrating the tight coupling existing between SiNW-bioFET design parameters affecting LoD and those related to noise injection and rejection at the instrumentation level. Although our analytical model is only that of a specific SiNW-bioFET/instrumentation instance and could not possibly be trusted quantitatively to predict the detection performances of another SiNWbioFET/instrument system, it illustrates the grand principles of sensor/instrument interactions and can be adapted to the specificities of various biological targets, sensor designs or instrumentation techniques.

The analytical model of the circular cross-section SiNWbioFET we have been using throughout this study is certainly over-simplifying as it does not translate the specific behaviours unique to each SiNW-bioFET design variant. Yet the generic nature of that model helps us make our point: irrespectively of the peculiar SiNW-bioFET design or operation mode, SiNW 
length does play a role on system-level LoD although studies on SiNW-bioFET alone do not predict that dependency (except if we consider short-channel effects but these are rarely a concern for junction-less bioFETs with channels at least a few micrometers long). The magnitude of the influence of SiNW length on LoD levels is itself function of the operation regime and dopant concentration of the SiNW: the higher the resistivity, the greater the systems LoD dependency on SiNW length. Along the same line of reasoning, it appears that the subthreshold regime, advocated for the high-sensitivity operation of SiNW-bioFETs may not always provide the best system-level LoD performances. SiNW-bioFET brought close to the subthreshold regime would indeed present very low intrinsic conductance, and impose the same stringent sensitivity and noise rejection requirements on the instrument as verypoorly doped SiNWs would. Figures 11d and 11c illustrate this apparent paradox: Scenarios where sensor baseline conductance is the lowest (figure 11d) provide better system LoDs for only a small subset of SiNW length/excitation frequency configurations whereas SiNWs presenting a higher baseline conductance provide consistent system LoD performances over the SiNW length/excitation frequency parameter space. The influence of the SiNW-bioFET operation regime was not precisely accounted for here as our analytical model does not allow for the specification of biasing conditions. Further work in that direction is therefore needed.

Also, we mentioned in our introduction that a SiNWbioFET/instrumentation system-level sensitivity analysis was desirable whenever a custom-, non-integrated instrumentation was to be coupled to a SiNW-bioFET array. In such a case it appears that if the design of a new SiNW-bioFET were to follow the design of its instrumentation, then the analytical noise- and signal processing model of the instrument ought to be taken into consideration when designing the sensor. In our case, the availability of a limited number of gains at the TIA stage should drive us to design SiNWs that are slightly longer than $7 \mathrm{~nm}$ if the dopant concentration is $1 \times 10^{24} \mathrm{~m}^{-3}$. There, baseline resistance of the sensor and optimal gain selection of the instrument would ensure optimal LoD performance.

Finally, our choice for the lock-in amplifier model may appear somewhat arbitrary if not for the fact that lock-in amplification is commonly the instrumentation of choice for SiNWbioFET investigations that do not rely on a silicon-integrated analog front end. Our investigation of the SiNW-bioFET/lockin amplifier coupling is therefore indicative of a few trade-offs system designers should be aware of. In particular, we showed that highly-resistive sensor configurations (e.g. low dopant concentrations, sub-threshold operation) narrowed down the range of usable excitation frequencies for the lock-in amplifier: too low and the LoD suffers from the injection of $1 / f$ noise; too high and the signal rolls-off beyond the TIA RC filter cutoff. This consideration can have important ramifications, for instance if continuous impedance monitoring is needed (e.g. for impedance-based cytometry, etc.) as TIA gains and operable frequency bandwidth are tighly coupled: if one is required to excite the sensor continuously at a high frequency then sharp FIR filters may not be selectable as they may not have time to complete their computations in real time. This would mean using less efficient FIR filters at the detriment of LoD performance, which may be an issue for spectroscopic applications looking at small signals. Conversely, high LoD performances may require the constriction of the excitation frequency to a very low bandwidth: spectroscopic applications could then be deprived of higher frequency measurements, which may impede their utility this time, or other performances, such as throughput [16].

\section{CONCLUSION}

The SiNW-bioFET technology is in many aspects promising for the detection of low concentration of biological markers in solution. We demonstrated that system-level considerations including both sensor and instrumentation may yet better inform on the performances one might expect from a complete sensor-instrumentation diagnostic system. We showed from such analyses that although the role of SiNW length and dopant concentration are well established when considered from a strict sensor perspective, they may need to be revisited when instrumentation is taken into account and if one wishes to leverage sensitive SiNW-bioFETs with an appropriately sensitive instrumentation or tailor the design of a novel SiNWbioFET if it is to be coupled to an existing instrument.

\section{APPENDIX A}

AN8608 VOLTAGE NOISE POWER SPECTRAL DENSITY

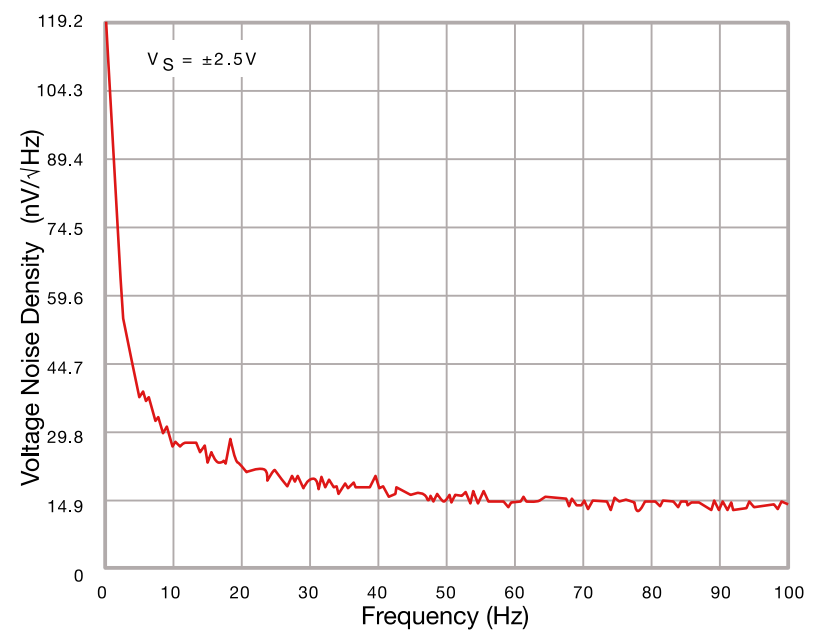

Fig. 12. Voltage noise spectral density of the AN8608 amplifier. The PSD shows the typical $1 / f$ dependency of many active electronic components at low frequency.

\section{APPENDIX B}

\section{CONTRIBUTION OF THE AMPLIFIER'S VOLTAGE NOISE}

Generally speaking, for a given frequency interval $\left[f_{L}-f_{H}\right]$, i.e. bandwidth, the expression of the TIA output RMS voltage noise $e_{v}$ is obtained from the integration of the amplifier's input NPSD from $f_{L}$ to $f_{H}$, taking into account the noise gain profile over this particular bandwidth [32]. If we consider a noise gain $N_{f}=1$, we obtain:

$$
e_{v}=\int_{f_{L}}^{f_{H}}\left(\mathrm{NPSD} \times N_{f}\right) \cdot d f=\int_{f_{L}}^{f_{H}} \mathrm{PSD} \cdot d f
$$


If the PSD spectrum is flat over the $f_{H}-f_{L}$ bandwidth, (white-noise spectrum) we have:

$$
e_{v}=\int_{f_{L}}^{f_{H}} C \cdot d f=C\left(f_{H}-f_{L}\right)
$$

Conversely if the PSD spectrum consists purely of $1 / f$ noise we get:

$$
e_{v}=\int_{f_{L}}^{f_{H}} \frac{K^{2}}{f} \cdot d f=K^{2} \ln \frac{f_{H}}{f_{L}}
$$

with $K^{2}$ a characteristic device constant.

Let us consider a noise PSD constituted of $1 / f$ noise for frequencies inferior to $f_{\mathrm{nc}}$, and displaying white noise for frequencies superior to $f_{\mathrm{nc}}$. We obtain $K^{2} / f_{\mathrm{nc}}=C$ and the expression of $e_{v}$ becomes:

$$
e_{v}=C\left(f_{\mathrm{nc}} \ln \frac{f_{H}}{f_{L}}+\left(f_{H}-f_{L}\right)\right)
$$

At $10 \mathrm{~Hz}$, the AN8608 input voltage noise PSD is $29.8 \times 10^{-9} \mathrm{~V} / \sqrt{\mathrm{H}} \mathrm{z}$. It can be shown that $K^{2}=((29.8 \times$ $\left.\left.10^{-9}\right)^{2}-e_{\text {high-freq }}^{2}\right) \times 10$ [38] and thus that $f_{\mathrm{nc}}=K^{2} / C^{2} \simeq$ $128 \mathrm{~Hz}$. We have $f_{\mathrm{nc}}<f_{i+f}$, meaning that the $1 / f$ noise components will be amplified by $N_{f}=1+R_{f} / R_{\text {sens }}$ which simplifies the calculations. We can segment the integral of equation 58 and derive the set of equivalence in (61).

$$
\left\{\begin{array}{l}
e_{v\left[0.01: f_{i+f}\right]}=8 \times 10^{-9}\left(1+\frac{R_{f}}{R_{\text {sens }}}\right) \sqrt{f_{\mathrm{nc}} \ln \left(\frac{f_{i+f}}{0.01}\right)+f_{i+f}-0.01}=8.848 \times 10^{-7} \mathrm{~V} \\
e_{v\left[f_{i+f}: f_{f}\right]}=8 \times 10^{-9}\left(\frac{1}{f_{i+f}}\right)\left(1+\frac{R_{f}}{R_{\text {sens }}}\right) \sqrt{\frac{f_{f}}{3}-\frac{f_{i+f}}{3}}=7.924 \times 10^{-11} \mathrm{~V} \\
e_{v\left[f_{f}:+\infty[\right.}=8 \times 10^{-9}\left(1+\frac{C_{i}}{C_{f}}\right) \sqrt{\left(\frac{\pi}{2} f_{\text {roll-off }}-f_{f}\right)}=8.861 \times 10^{-6} \mathrm{~V}
\end{array}\right.
$$

We arbitrarily chose a lower bound for the first segment at $f=0.01 \mathrm{~Hz}$ as the integral converges when $f$ tends towards $0 \mathrm{~Hz}$. The third segment is unbounded at high-frequencies i.e. $f_{H}=+\infty$, but we know that the noise gain profile limits system bandwidth beyond $f_{\text {roll-off }}=1.3 \times 10^{6} \mathrm{MHz}$ with a $-6 \mathrm{~dB}$ octave $^{-1}$ single-pole filter roll-off. The contribution of the attenuated noise components beyond that cut-off are incorporated by using by the expression of the Equivalent Noise Bandwidth (ENBW) of a single-pole filter for this segment [32], giving:

$$
\mathrm{ENBW}=\frac{\pi}{2}\left(f_{\text {roll-off }}-f_{f}\right) \mathrm{Hz}
$$

The total TIA RMS output noise $e_{v}$ attributable to $e_{n}$ is then:

$$
\begin{aligned}
& e_{v}=\sqrt{\left(e_{v\left[0.01: f_{i+f}\right]}\right)^{2}+\left(e_{v\left[f_{i+f}: f_{f}\right]}\right)^{2}+\left(e_{v\left[f_{f}:+\infty[\right.}\right)^{2}} \\
& e_{v} \simeq 8.905 \times 10^{-6} \mathrm{~V}_{\mathrm{rms}}
\end{aligned}
$$

$$
\left\{\begin{array}{l}
e_{i\left[0: f_{i+f}\right]}=1 \times 10^{-14}\left(R_{f}+R_{b}\left(1+\frac{R_{f}}{R_{\text {sens }}}\right)\right) f_{i+f}=3.807 \times 10^{-4} \mathrm{~V} \\
e_{i\left[f_{i+f}: f_{f}\right]}=1 \times 10^{-14}\left(R_{f}+R_{b}\left(1+\frac{R_{f}}{R_{\text {sens }}}\right)\left(\frac{1}{f_{i+f}}\right)\right) \sqrt{\frac{f_{f}}{3}-\frac{f_{i+f}}{3}}=4.762 \times 10^{-5} \mathrm{~V} \\
\left.e_{i\left[f_{f}:+\infty[\right.}=1 \times 10^{-14}\left(R_{f}+R_{b}\left(1+\frac{C_{i}}{C_{f}}\right)\right) \sqrt{\frac{\pi}{2}\left(f_{\text {roll-off }}-f_{f}\right.}\right)=1.3197 \times 10^{-3} \mathrm{~V}
\end{array}\right.
$$

We can conclude:

$$
\begin{aligned}
& e_{i}=\sqrt{\left(e_{i\left[0: f_{i+f}\right]}\right)^{2}+\left(e_{i\left[f_{i+f}: f_{f}\right]}\right)^{2}+\left(e_{i\left[f_{f}:+\infty\right.}[)^{2}\right.} \\
& e_{i} \simeq 1.3744 \times 10^{-3} \mathrm{~V}_{\mathrm{rms}}
\end{aligned}
$$




\section{APPENDIX D}

CONTRIBUTION OF RB

$$
\left\{\begin{array}{l}
e_{R_{b}\left[0: f_{i+f}\right]}=\sqrt{4 k_{B} T R_{b}\left(1+\frac{R_{f}}{R_{\text {sens }}}\right)^{2} f_{i+f}}=7.542 \times 10^{-7} \mathrm{~V} \\
e_{R_{b}\left[f_{i+f}: f_{f}\right]}=\sqrt{4 k_{B} T R_{b}\left(1+\frac{R_{f}}{R_{\text {sens }}}\right)^{2}\left(\frac{1}{f_{i+f}}\right)^{2}\left(\frac{f_{f}}{3}-\frac{f_{i+f}}{3}\right)}=1.529 \times 10^{-8} \mathrm{~V} \\
e_{R_{b}\left[f_{f}:+\infty[\right.}=\sqrt{4 k_{B} T R_{b}\left(1+\frac{C_{i}}{C_{f}}\right)^{2}\left(\frac{\pi}{2}\left(f_{\text {roll-off }}-f_{f}\right)\right)}=4.493 \times 10^{-5} \mathrm{~V}
\end{array}\right.
$$

\section{APPENDIX E}

DEMODULATED FILTERED NOISE CONTRIBUTIONS

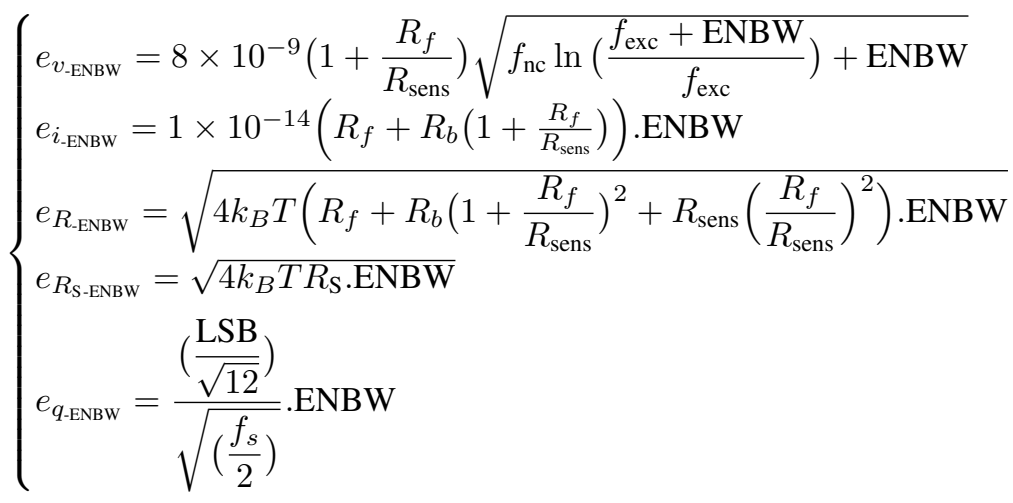

Note that the distribution for the quantization noise power spectral density is assumed to be uniform over the entire ADC bandwidth $f_{s} / 2$ [35].

The expression of $V_{2 . f_{\text {exc }} \text { rms }}$ corresponds to the FIR filtered harmonic at 2. $f_{\mathrm{exc}}$. As discussed previously, this harmonic corresponds to half the energy of the main harmonic signal at the input of the ADC, taking into account the possible attenuations/gain throughout the signal conditioning block. We can thus derive :

$$
\begin{aligned}
& V_{\left(2 f_{\text {exc }}\right) \mathrm{rms}}=\frac{V_{\text {demod }_{\text {out }}}\left(2 \cdot f_{\text {exc }}\right) \cdot\left|H_{\mathrm{FIR}}\left(2 \cdot f_{\mathrm{exc}}\right)\right|}{\sqrt{2}} \\
& V_{\left(2 f_{\text {ex }}\right) \mathrm{rms}}=\frac{V_{\text {exc }} \cdot\left|H_{\mathrm{TIA}}\left(f_{\text {exc }}\right)\right| \cdot\left|H_{\text {anti-alias }}\left(f_{\text {exc }}\right)\right| \cdot\left|H_{\mathrm{FIR}}\left(2 \cdot f_{\text {exc }}\right)\right|}{Z_{\text {sens }} 2 \sqrt{2}}
\end{aligned}
$$

For this specific case $\left(f_{\mathrm{exc}}=10 \mathrm{~Hz}\right)$, the expression of the $e_{\mathrm{PSD}_{\text {out }}}$ gives:

$$
\begin{aligned}
e_{\mathrm{PSD}_{\text {out }}} & =\left(e_{v_{\mathrm{ENBW}}}^{2}+e_{i_{\mathrm{ENBW}}}^{2}+e_{R_{\mathrm{ENBW}}}^{2}+e_{R_{\mathrm{S}} \mathrm{ENBW}}^{2}\right. \\
& \left.+e_{q_{\mathrm{ENBW}}^{2}}^{2}\right)^{1 / 2}+V_{\left(2 f_{\text {exc }}\right) \mathrm{rms}} \\
e_{\mathrm{PSD}_{\text {out }}} & =\left(1.533 \times 10^{-13}+4.483 \times 10^{-9}\right. \\
& +2.222 \times 10^{-9}+7.373 \times 10^{-16} \\
& \left.\left.+1.212 \times 10^{-7}\right)^{1 / 2}+1.826 \times 10^{-3}\right\} \\
e_{\mathrm{PSD}_{\text {out }}} & =2.184 \times 10^{-3} \mathrm{~V}
\end{aligned}
$$

\section{APPENDIX F}

FIR FILTER BANK

ACKNOWLEDGMENT

This project is a part of the EU Marie Curie Initial Training Networks (ITN) Biomedical Engineering for Cancer and BRAin disease diagnosis and therapy development: EngCaBra ; Project No. PITN-GA-2010-264417. 


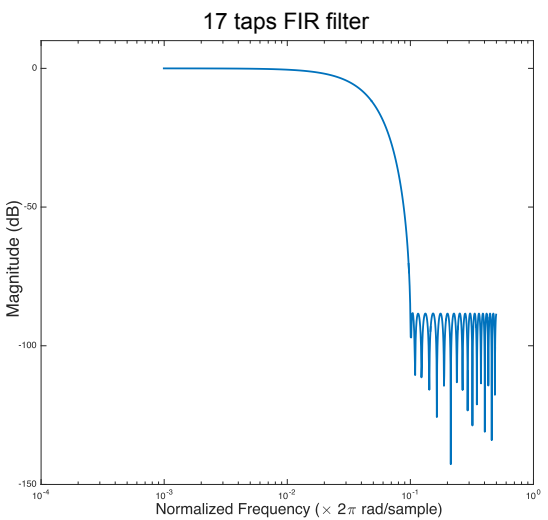

(a)

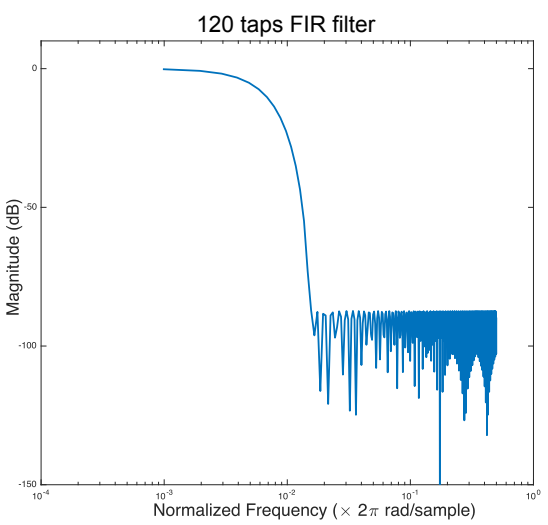

(d)

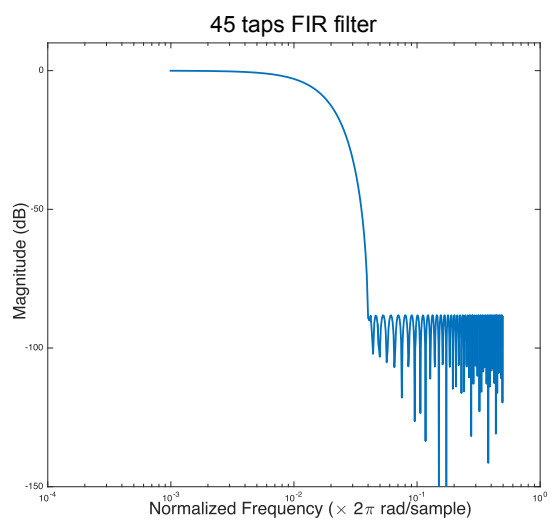

(b)

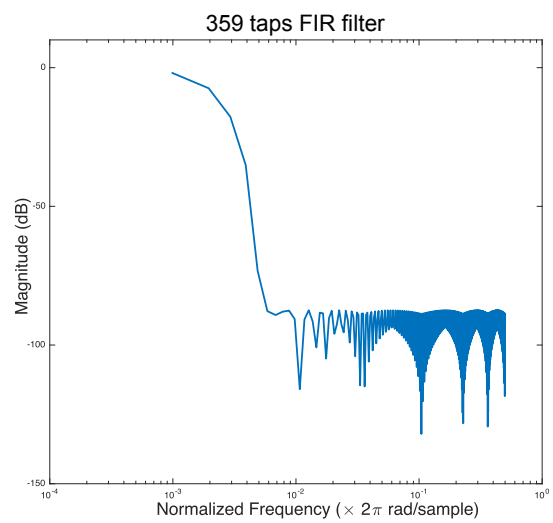

(e)

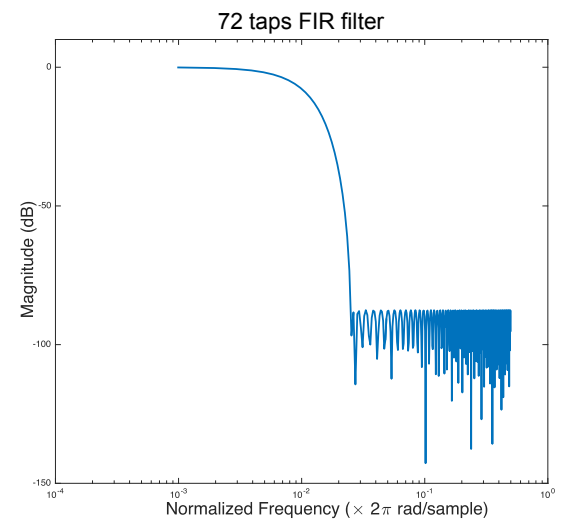

(c)

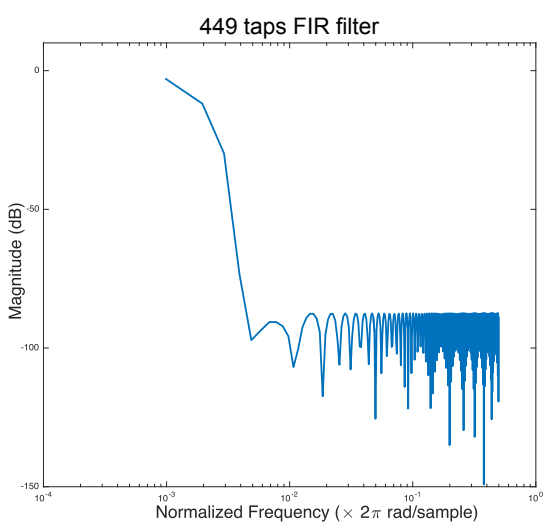

(f)

Fig. 13. FIR filter bank frequency response

\section{REFERENCES}

[1] Y. Cui, Q. Wei, H. Park, and C. M. Lieber, "Nanowire nanosensors for highly sensitive and selective detection of biological and chemical species." Science (New York, N.Y.), vol. 293, no. 5533, pp. 1289-1292, 2001.

[2] M. Wipf, R. L. Stoop, A. Tarasov, K. Bedner, W. Fu, I. A. Wright, C. J. Martin, E. C. Constable, M. Calame, and C. Schönenberger, "Selective Sodium Sensing with Gold-Coated Silicon Nanowire Field-Effect Transistors in a Differential Setup," ACS Nano, vol. 7, no. 7, pp. 59785983, jul 2013. [Online]. Available: http://dx.doi.org/10.1021/nn401678u

[3] J.-i. Hahm and C. M. Lieber, "Direct Ultrasensitive Electrical Detection of DNA and DNA Sequence Variations Using Nanowire Nanosensors," Nano Letters, vol. 4, no. 1, pp. 51-54, 2004. [Online]. Available: http://pubs.acs.org/doi/abs/10.1021/n1034853b

[4] G. J. Zhang, J. H. Chua, R. E. Chee, A. Agarwal, and S. M. Wong, "Label-free direct detection of MiRNAs with silicon nanowire biosensors," Biosensors and Bioelectronics, vol. 24, no. 8, pp. 2504-2508, 2009.

[5] Y. L. Wu, J. J. Lin, P. Y. Hsu, and C. P. Hsu, "Highly sensitive polysilicon wire sensor for DNA detection using silica nanoparticles/??-APTES nanocomposite for surface modification," Sensors and Actuators, B: Chemical, vol. 155, no. 2, pp. 709-715, 2011. [Online]. Available: http://dx.doi.org/10.1016/j.snb.2011.01.035

[6] A. Kim, C. S. Ah, H. Y. Yu, J. H. Yang, I. B. Baek, C. G. Ahn, C. W. Park, M. S. Jun, and S. Lee, "Ultrasensitive, label-free, and real-time immunodetection using silicon field-effect transistors," Applied Physics Letters, vol. 91, no. 10, 2007.

[7] F. Patolsky, G. Zheng, O. Hayden, M. Lakadamyali, X. Zhuang, and C. M. Lieber, "Electrical detection of single viruses." Proceedings of the National Academy of Sciences of the United States of America, vol. 101, no. 39, pp. 14017-14022, 2004.

[8] G.-J. Zhang, L. Zhang, M. J. Huang, Z. H. H. Luo, G. K. I. Tay, E.-J. A. Lim, T. G. Kang, and Y. Chen, "Silicon nanowire biosensor for highly sensitive and rapid detection of Dengue virus," Sensors and
Actuators B: Chemical, vol. 146, no. 1, pp. 138-144, 2010. [Online]. Available: http://dx.doi.org/10.1016/j.snb.2010.02.021

[9] S. Regonda, R. Tian, J. Gao, S. Greene, J. Ding, and W. Hu, "Silicon multi-nanochannel FETs to improve device uniformity/stability and femtomolar detection of insulin in serum," Biosensors and Bioelectronics, vol. 45, no. 1, pp. 245-251, 2013. [Online]. Available: http://dx.doi.org/10.1016/j.bios.2013.01.027

[10] F. Puppo, M.-A. Doucey, T. S. Y. Moh, G. Pandraud, P. M. Sarro, G. De Micheli, and S. Carrara, "Femto-molar sensitive field effect transistor biosensors based on silicon nanowires and antibodies," in SENSORS IEEE 2013, 2013, pp. 1-4.

[11] F. Puppo, M.-A. Doucey, J.-F. Delaloye, T. S. Y. Moh, G. Pandraud, P. M. Sarro, G. De Micheli, and S. Carrara, "SiNW-FET in-Air Biosensors for High Sensitive and Specific Detection in Breast Tumor Extract," IEEE Sensors Journal, vol. 16, no. 10, pp. 3374-3381, 2016.

[12] X. P. a. Gao, G. Zheng, and C. M. Lieber, "Subthreshold regime has the optimal sensitivity for nanowire FET biosensors," Nano Letters, vol. 10, no. 2, pp. 547-552, 2010.

[13] L. De Vico, M. H. Sørensen, L. Iversen, D. M. Rogers, B. S. Sørensen, M. Brandbyge, J. Nygård, K. L. Martinez, and J. H. Jensen, "Quantifying signal changes in nano-wire based biosensors." Nanoscale, vol. 3, no. 2, pp. 706-717, 2011.

[14] F. Patou, F. Alatraktchi, C. Kjægaard, M. Dimaki, J. Madsen, and W. E. Svendsen, "Evolvable Smartphone-Based Platforms for Point-Of-Care In-Vitro Diagnostics Applications," Diagnostics, vol. 6, no. 3, pp. 1-18, 2016.

[15] J. Lee, J. Jang, B. Choi, J. Yoon, J.-Y. Kim, Y.-K. Choi, D. Myong Kim, D. Hwan Kim, and S.-J. Choi, "A Highly Responsive Silicon Nanowire/Amplifier MOSFET Hybrid Biosensor," Scientific Reports, vol. 5, no. 1, p. 12286, 2015.

[16] A. Susloparova, D. Koppenh, X. T. Vu, M. Weil, and S. Ingebrandt "Author' s personal copy Biosensors and Bioelectronics Impedance spectroscopy with field-effect transistor arrays for the analysis of anticancer drug action on individual cells \$," vol. 40, pp. 50-56, 2013.

[17] F. Puppo, F. L. Traversa, M. Di Ventra, G. De Micheli, and S. Carrara, 
"Surface trap mediated electronic transport in biofunctionalized silicon nanowires," Nanotechnology, vol. 27, 2016.

[18] E. Stern, R. Wagner, F. J. Sigworth, R. Breaker, T. M. Fahmy, and M. a Reed, "Importance of the debye screening length on nanowire field effect transistor sensors," Nano Letters, vol. 7, no. 11, pp. 3405-3409, 2007.

[19] P. R. Nair and M. a. Alam, "Performance limits of nanobiosensors," Applied Physics Letters, vol. 88, no. 23, 2006.

[20] P. R. Nair, S. Member, and M. a. Alam, "Design Considerations of Silicon Nanowire Biosensors," IEEE Transactions on Electron Devices, vol. 54, no. 12, pp. 3400-3408, 2007.

[21] N. Elfström, A. E. Karlström, and J. Linnros, "Silicon nanoribbons for electrical detection of biomolecules," Nano Letters, vol. 8, no. 3, pp. 945-949, 2008.

[22] P. R. Nair and M. a. Alam, "Screening-limited response of NanoBiosensors," Nano Letters, vol. 8, no. 5, pp. 1281-1285, 2008.

[23] L. De Vico, M. H. Sørensen, L. Iversen, D. M. Rogers, B. S. Sørensen, M. Brandbyge, J. Nygård, K. L. Martinez, and J. H. Jensen, "Quantifying signal changes in nano-wire based biosensors." Nanoscale, vol. 3, no. 2, pp. 706-717, 2011.

[24] E. Buitrago, G. Fagas, M. F. B. Badia, Y. M. Georgiev, M. Berthomé, and A. M. Ionescu, "Junctionless silicon nanowire transistors for the tunable operation of a highly sensitive, low power sensor," Sensors and Actuators, B: Chemical, vol. 183, pp. 1-10, 2013.

[25] N. Elfström, Silicon Nanowires for Biomolecule Detection, 2008. [Online]. Available: http://en.scientificcommons.org/344448898

[26] Y. L. Bunimovich, Y. S. Shin, W. S. Yeo, M. Amori, G. Kwong, and J. R. Heath, "Quantitative real-time measurements of DNA hybridization with alkylated nonoxidized silicon nanowires in electrolyte solution," Journal of the American Chemical Society, vol. 128, no. 50, pp. 16323-16331, 2006.

[27] M. Y. Shen, B. R. Li, and Y. K. Li, "Silicon nanowire fieldeffect-transistor based biosensors: From sensitive to ultra-sensitive," Biosensors and Bioelectronics, vol. 60, pp. 101-111, 2014. [Online]. Available: http://dx.doi.org/10.1016/j.bios.2014.03.057

[28] M. H. Sørensen, N. A. Mortensen, and M. Brandbyge, "Screening model for nanowire surface-charge sensors in liquid," Applied Physics Letters, vol. 91 , no. 10, 2007

[29] G. Ferrari, F. Gozzini, and M. Sampietro, "Transimpedance amplifiers for extremely high sensitivity impedance measurements on nanodevices," Analog Circuit Design: High-speed Clock and Data Recovery, High-performance Amplifiers, Power Management, pp. 193-207, 2006.

[30] A. Device, "Analog Devices AN8608 Data Sheet," pp. 1-24, 2003.

[31] L. Orozco, "Programmable-Gain Transimpedance Amplifiers Maximize Dynamic Range in Spectroscopy Systems," Analog Dialogue, vol. 47, no. 05 , pp. $1-5,2013$.

[32] A. devices, "MT-048 - Op Amp Noise Relationships: 1/f Noise, RMS Noise, and Equivalent Noise Bandwidth," Tech. Rep., 2010.

[33] S. Reza, G. Bosman, M. S. Islam, T. I. Kamins, S. Sharma, and R. S. Williams, "Noise in silicon nanowires," IEEE Transactions on Nanotechnology, vol. 5, no. 5, pp. 523-528, 2006.

[34] N. K. Rajan, D. A. Routenberg, J. Chen, and M. A. Reed, "1/f noise of silicon nanowire BioFETs," IEEE Electron Device Letters, vol. 31 , no. 6, pp. 615-617, 2010.

[35] W. Kester, "Taking the Mystery out of the Infamous Formula," SNR $=6.02 \mathrm{~N}+1.76 \mathrm{~dB}$, and Why You Should Care," MT-001 Tutorial, pp. 1-7, 2005. [Online]. Available: http://qt.tn.tudelft.nl/ schouten/linkload/adc-tutorial.pdf

[36] M. L. Meade, Lock-in Amplifiers: Principles and Applications, ser IEE electrical measurement series. P. Peregrinus, 1983. [Online]. Available: https://books.google.dk/books?id=cg9TAAAAMAAJ

[37] J. Leis, C. Kelly, and D. Buttsworth, "Sampling, Quantization and Computational Aspects of the Quadrature Lock-In Amplifier," in Signal Processing and Communication Systems (ICSPCS), 2012 6th International Conference on, 2012, pp. 1-7.

[38] Texas Instruments, "Noise Analysis in Operational Amplifier Circuits," Tech. Rep., 2007.

François Patou Frano̧is Patou holds an M.Sc from the Technical University of Compiegne (UTC, France) in Biomedical Engineering, and completed his Ph.D in 2016, which he pursued in collaboration with the Department of Micro- and Nanotechnology and the Department of Applied Mathematics and Computer Science at the Technical University of Denmark (DTU). Franois has carried out research on the design of evolvable medical and life-science technological systems. He is currently a Post-Doctoral researcher at the Engineering Systems Division within the Department of Management Engineering at DTU where he focuses on the integration of wearable technologies in healthcare practices.

Maria Dimaki Maria Dimaki holds a Ph.D from the Department of Microand Nanotechnology at the Technical University of Denmark, where she received her degree in 2005 after having worked with the assembly of carbon nanotube devices using dielectrophoresis. Dr. Dimaki has remained with the department since then, and is currently a senior researcher within the NanoBio Integrated Systems group (NaBIS). Her research interests mainly involve the development of sensors for biological and environmental applications. This includes the design and fabrication of the sensors in a cleanroom, as well as the design, numerical simulations and fabrication of the microfluidic networks that deliver the sample to the sensors.

Claus Kjægaard Claus Kjægaard is Professor at DTU Diplom, Center for B.Eng. at DTU Ballerup campus at the Technical University of Denmark (DTU). For many years his research was focused on reliability of repairable electronic systems. During the last 10 years his work has been focused on teaching and developing courses and study plans in the field of analog electronics and hybrid microelectronics and on research in these fields. In 2006 he received the reward as best teacher in the B.Eng. educational programs at DTU. Since 2005 he has been head of studies for the B.Eng. program in electronics.

Jan Madsen Jan Madsen is Full Professor in Computer-Based Systems at Department of Applied Mathematics and Computer Science (DTU Compute), Technical University of Denmark (DTU). He is working on design, modeling, analysis, optimization and construction of microelectronic, microfluidic and microbiological computing systems. He is heading a research group on Embedded Systems Engineering and is Deputy Director of DTU Compute. He has published more than 150 peer-reviewed conference and journal papers, 12 book chapters, and 3 books. Since 2007, he has been part of a research group focusing on Design Automation for biochips. Since 2011 he has coordinated research on synthetic biology with the vision to link it to the biochip research. He holds 2 patents on a bio-inspired self-healing computer architecture from which he has co-founded the spin-off company Biomicore.

Winnie E. Svendsen Winnie E. Svendsen received her MSc degree in physics in 1993 from the University College Dublin, Ireland; there, she received the EOLAS applied research award for excellent research. She obtained a PhD from Copenhagen University and the National Laboratory for Sustainable Energy in 1996. She then accepted a postdoctoral position at the Max Planck Institute for plasma physics. In 1998 Dr. Svendsen received talent stipend from SNF (now FNU) and the prestigious Curie stipend from Copenhagen University to establish a research group to design a hyperpolarized gas setup for use in medical lung diagnostic. In connection with this project she received funding from the European Fifth Framework to organize a workshop on hyperpolarized gasses. In 1999 she was appointed associate professor at Copenhagen University. Since 2000 Dr. Svendsen has been employed as associated professor at DTU. In 2006 she established her own research group, Nano-Bio Integrated Systems (NaBIS), which works on the development of integrated sensors for biological and environmental application 